\title{
Power and bipower variation with stochastic volatility and jumps
}

\author{
Ole E. BARNDORFF-NIELSEN \\ The Centre for Mathematical Physics and Stochastics (MaPhySto), \\ University of Aarhus, Ny Munkegade, DK-8000 Aarhus C, Denmark. \\ oebn@mi . aau. dk \\ http://home.imf.au.dk/oebn/ \\ NeIL SHEPHARD \\ Nuffield College, University of Oxford, Oxford OX1 1NF, UK \\ neil.shephard@nuf.ox.ac.uk \\ www.nuff.ox.ac.uk/users/shephard/
}

This draft: 2nd November 2003

\begin{abstract}
This paper shows that realised power variation and its extension called realised bipower variation that we introduce here is somewhat robust to rare jumps. We demonstrate that in special cases realised bipower variation estimate integrated variance in stochastic volatility models, thus providing a model free and consistent alternative to realised variance. Its robustness property means that if we have a stochastic volatility plus infrequent jumps process then the difference between realised variance and realised bipower variation estimates the quadratic variation of the jump component. This seems to be the first method which can separate quadratic variation into its continuous and jump components. Various extensions are given, together with proofs of special cases of these results. Detailed mathematical results will be reported elsewhere.

Keywords: Bipower variation; Integrated variance; Jump process; Power variation; Quadratic variation; Realised variance; Realised volatility; Semimartingale; Volatility.
\end{abstract}

\section{Introduction}

\subsection{Motivation}

The econometrics of financial volatility has made very significant recent progress due to the harnessing of high frequency information through the use of realised variances. Here we discuss generalisations of objects of that type, studying sums of powers and products of powers of absolute returns. We will show that these quantities, called realised power variation and bipower variation, respectively, are both quite robust to rare jumps in the log-price process. In particular we demonstrate that it is possible, in theory, to untangle the impact of the presence of volatility and rare jumps by using power and bipower variation. Realised bipower variation also provides a new asymptotically unbiased, model free econometric estimator of integrated variance in stochastic volatility (SV) models. This estimator is robust to the presence of jumps. 
Hence, in theory, we can now decompose quadratic variation into the contribution from the continuous component of log-prices and the impact of jumps. Given that quadratic variation plays a crucial role in the theory of measuring the risk associated with an asset, being able to separate the diffusion and jump risks seems interesting. We believe ours is the first paper to do this without making strong parametric assumptions.

To start, let us establish some notation. Suppose that $\hbar>0$ is some fixed time period (e.g. a trading day or a calendar month) and that the log-price of an asset is written as $y^{*}(t)$ for $t \geq 0$. Then the $i$-th $\hbar$ "low frequency" return is

$$
y_{i}=y^{*}(i \hbar)-y^{*}((i-1) \hbar), \quad i=1,2, \ldots .
$$

For concreteness we will often refer to the $i$-th period as the $i$-th day. Suppose, in addition to $y_{i}$, that we record the prices at $M$ equally spaced time points during the $i$-th day. Then we can define the "high frequency" returns as

$$
y_{j, i}=y^{*}\left((i-1) \hbar+\hbar j M^{-1}\right)-y^{*}\left((i-1) \hbar+\hbar(j-1) M^{-1}\right), \quad j=1,2, \ldots, M .
$$

Here $y_{j, i}$ is the $j$-th intra- $\hbar$ return for the $i$-th day (e.g. if $M=288$, then this is the return for the $j$-th 5 minute period on the $i$-th day). As a result, for example, $y_{i}=\sum_{j=1}^{M} y_{j, i}$.

To illustrate this notation we will look at the first three days of the Olsen and Associates' dollar/DM high frequency series. It starts on 1st December 1986 and ignores weekend breaks. This series is constructed every five minutes by the Olsen group from bid and ask quotes which appeared on the Reuters screen (see Dacorogna, Gencay, Müller, Olsen, and Pictet (2001) for details). The log values of the raw data, having being preprocessed in a manner discussed in the Appendix of Barndorff-Nielsen and Shephard (2002b), are shown in the top left of Figure 1. We have shifted the data to start at zero at time zero. This series constitutes an empirical counterpart, recorded every five minutes, to our $y^{*}$ process. The top right part of the Figure shows the first three daily returns $y_{1, i}=y_{i}$, i.e. $M=1$, calculated off this series. Hence in this case $i=1,2, \ldots, 3$. These returns are shown as large squares. The bottom left part shows $y_{j, i}$, for $j=1,2, \ldots, 8$ and $i=1,2,3$. These are 3 hour returns. Finally, the bottom right graph shows the effect of taking $M=48$, which corresponds to 30 minute returns.

In this paper we begin by recalling the well known connections between realised variance

$$
\left\{y_{M}^{*}\right\}_{i}^{[2]}=\left[y_{M}^{*}\right]_{i}^{[2]}=\sum_{j=1}^{M} y_{j, i}^{2},
$$




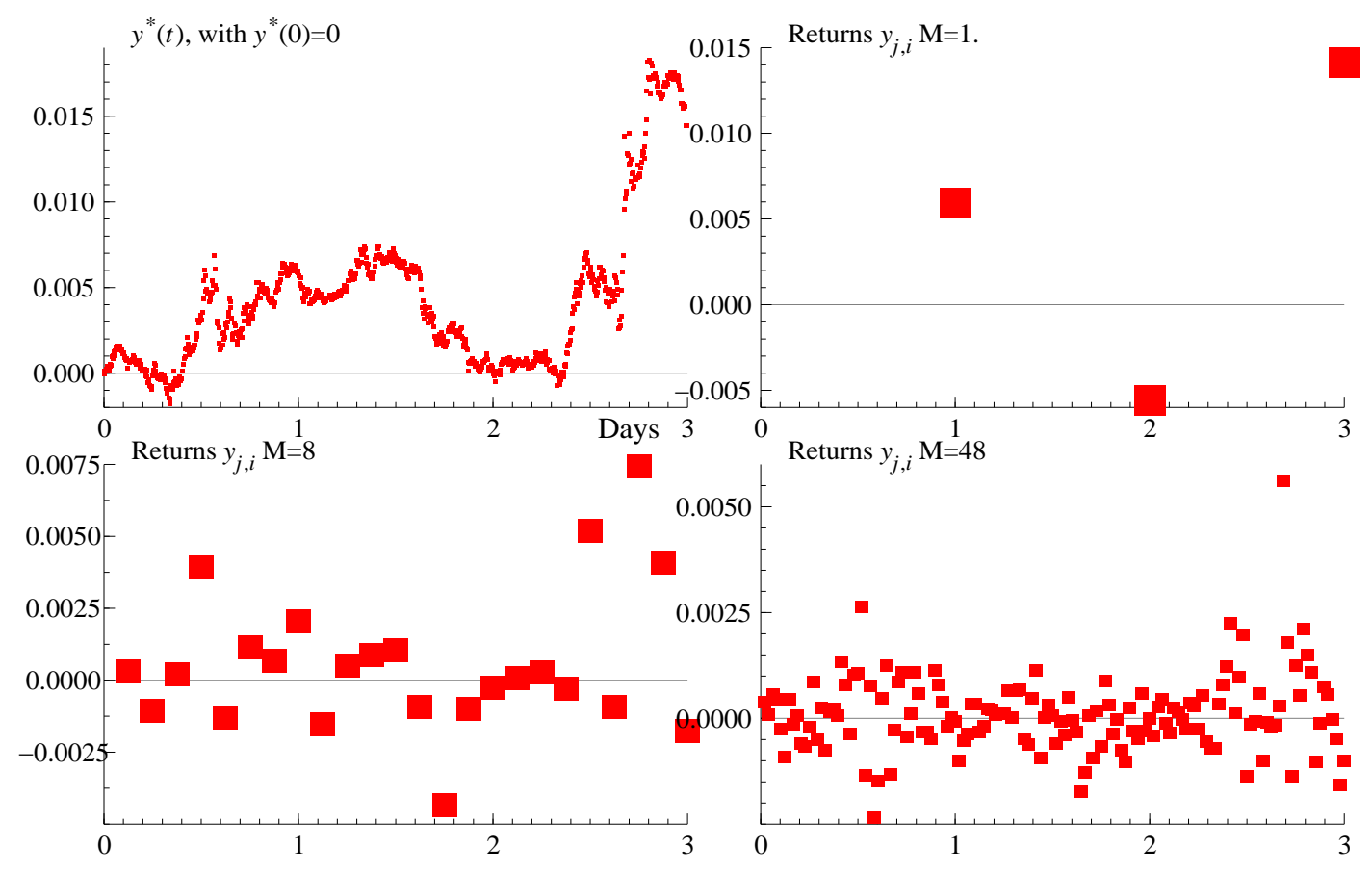

Figure 1: DM/dollar dataset from the Olsen and Associates database. Top left: raw 5 minute data. Top right: daily returns, $M=1$. Bottom left: 3 hour returns, $M=8$. Bottom right: 30 minute returns, $M=48$.

and quadratic variation ${ }^{1}$. This is also linked to its square root version ${ }^{2}$, the realised volatility $\sqrt{\sum_{j=1}^{M} y_{j, i}^{2}}$. The paper then progresses to work with more general aggregate volatility measures. These will initially be based on realised power variation

$$
\left\{y_{M}^{*}\right\}_{i}^{[r]}=\left(\frac{\hbar}{M}\right)^{1-r / 2} \sum_{j=1}^{M}\left|y_{j, i}\right|^{r}, \quad r>0
$$

and, to a lesser extent, its unnormalised version ${ }^{3}\left[y_{M}^{*}\right]_{i}^{[r]}=\sum_{j=1}^{M}\left|y_{j, i}\right|^{r}$. These quantities have recently been formalised by Barndorff-Nielsen and Shephard (2003d). A review of some of the work on this topic is given in Barndorff-Nielsen, Graversen, and Shephard (2003). When $r=2$

\footnotetext{
${ }^{1}$ Realised variances have been used for a long time in financial economics — see, for example, Poterba and Summers (1986), Schwert (1989) and Dacorogna, Müller, Olsen, and Pictet (1998). Realised variance has been studied from a methodological viewpoint by, for example, Andersen, Bollerslev, Diebold, and Labys (2001), Barndorff-Nielsen and Shephard (2001) and Barndorff-Nielsen and Shephard (2002b). See Andersen, Bollerslev, and Diebold (2004) for a survey of this area, including a discussion of the related literature.

${ }^{2}$ Note that in econometrics sums of squared returns are sometimes called realised volatility.

${ }^{3}$ The similarly named $p$-variation, $0<p<\infty$, of a real-valued function $f$ on $[a, b]$ is defined as

$$
\sup _{\kappa} \sum\left|f\left(x_{i}\right)-f\left(x_{i-1}\right)\right|^{p}
$$

where the supremum is taken over all subdivisions $\kappa$ of $[a, b]$. If this function is finite then $f$ is said to have bounded p-variation on $[a, b]$. The case of $p=1$ gives the usual definition of bounded variation. This concept of p-variation has been studied recently in the probability literature. See the work of Lyons (1994), Mikosch and Norvaiša (2000) and Lyons and Qian (2002).
} 
this yields the traditional realised variance. When $r=1$ we produce realised absolute variation ${ }^{4}$ $\left\{y_{M}^{*}\right\}_{i}^{[1]}=\sqrt{\frac{\hbar}{M}} \sum_{j=1}^{M}\left|y_{j, i}\right|$.

The most important contribution of this paper is the introduction of a generalisation of the above ideas. This is called realised bipower variation

$$
\left\{y_{M}^{*}\right\}_{i}^{[r, s]}=\left\{\left(\frac{\hbar}{M}\right)^{1-(r+s) / 2}\right\} \sum_{j=1}^{M-1}\left|y_{j, i}\right|^{r}\left|y_{j+1, i}\right|^{s}, \quad r, s \geq 0 .
$$

In particular, for example, we will show that in the $r=s=1$ case

$$
\left\{y_{M}^{*}\right\}_{i}^{[1,1]}=\sum_{j=1}^{M-1}\left|y_{j, i}\right|\left|y_{j+1, i}\right|
$$

will, up to a simple known multiple, converge to the same probability limit as realised variance when prices follow a SV process and that for (3) the limit does not change with the addition of rare jumps. This provides a new way of making inference and predicting integrated variance in SV models, perhaps the single most important term in the econometrics of volatility. Further, it gives a simple way of measuring the impact of jumps on quadratic variation.

\subsection{Outline of the paper}

In Section 2 of this paper we review the well known probability limit of realised variance, which is built on the theory of semimartingales and the quadratic variation process. We specialise the theory to a class of continuous sample path stochastic volatility semimartingales. For this class it is possible to extend the quadratic variation process to the power variation process, which allows us to derive the probability limit of realised power variation. Finally, we introduce the idea of bipower variation and study some of its properties.

Having established the probability limit for the various realised variations, in Section 3 we find the probability limit of realised power variation in the case where we add a compound Poisson process to the SV process. We see that sometimes the limit is not changed by the addition of jumps. We then extend this analysis to realised bipower variation and see that the robustness result holds so long as the maximum of the powers is less than two. This result is the main contribution of the paper.

\footnotetext{
${ }^{4}$ Andersen and Bollerslev (1998b) and Andersen and Bollerslev (1997) empirically studied the properties of sums of absolute values of intra-day returns on speculative assets. This was empirically attractive, for using absolute values is less sensitive to possible large movements in high frequency data. However, the approach was abandoned in their subsequent work reported in Andersen and Bollerslev (1998a) and Andersen, Bollerslev, Diebold, and Labys (2001) due to the lack of appropriate theory for the sum of absolute returns as $M \rightarrow \infty$, although recently Andreou and Ghysels (2002) have performed some interesting Monte Carlo studies in this context, while Shiryaev (1999, pp. 349-350) and Maheswaran and Sims (1993) mention interest in the limit of sums of absolute returns.
} 
In Section 4 we conduct a simulation study of a SV plus jump process, demonstrating that the theory seems to yield useful predictions. In Section 5 we apply the theory to some empirical data and in Section 6 we indicate various possible extensions of our work. Section 7 concludes.

\section{Basic development and ideas}

\subsection{Realised variance and quadratic variation}

The $i$-th realised variance is based on squares of high frequency observations in the interval of time between $\hbar(i-1)$ and $\hbar i$. To understand the properties of realised variance we need to connect it to the quadratic variation process - which we will define in a moment. It is traditional, convenient and elegant to write the theory of quadratic variation based on the interval from time 0 to $t$. We will follow that route here and continue with it when we initially discuss its generalisations to power variation. We can then back out the implication for items such as realised variance, which are based solely on quantities calculated on the interval from $\hbar(i-1)$ to $\hbar i$. These specific realised variance results will be presented at the end of this subsection.

The probability limit of realised variance (1) is known under the assumption that $y^{*}$ is a semimartingale $(\mathcal{S M})$, using the theory of quadratic variation (e.g. Jacod and Shiryaev (1987, p. 55)). Recall if $y^{*} \in \mathcal{S M}$, then we can write

$$
y^{*}=\alpha^{*}+m^{*}
$$

where $\alpha^{*}$, a drift term, has locally finite variation paths $\left(\mathcal{F} \mathcal{V}_{l o c}\right)$ and $m^{*}$ is a local martingale $\left(\mathcal{M}_{\text {loc }}\right)$. One of the most important aspects of semimartingales is the quadratic variation (QV) process. This is defined as

$$
\left[y^{*}\right](t)=\mathrm{p}-\lim _{M \rightarrow \infty} \sum_{j=1}^{M}\left\{y^{*}\left(t_{j}\right)-y^{*}\left(t_{j-1}\right)\right\}^{2},
$$

for any sequence of partitions $t_{0}=0<t_{1}<\ldots<t_{M}=t$ so long as $\sup _{j}\left\{t_{j}-t_{j-1}\right\} \rightarrow 0$ for $M \rightarrow \infty$.

In general

$$
\begin{aligned}
{\left[y^{*}\right](t) } & =\left[y^{* c}\right](t)+\left[y^{* d}\right](t) \\
& =\left[y^{* c}\right](t)+\sum_{0 \leq s \leq t}\left\{\Delta y^{*}(s)\right\}^{2},
\end{aligned}
$$

where $y^{* c}$ is the continuous local martingale component of $y^{*}, y^{* d}$ the corresponding jump process and $\Delta y^{*}(t)=y^{*}(t)-y^{*}(t-)$ is the jump at time $t$. If $\alpha^{*} \in \mathcal{F} \mathcal{V}_{l o c}^{c}$ (i.e. the subset of the $\mathcal{F} \mathcal{V}_{\text {loc }}$ class that has continuous sample paths) then we obtain the simplification

$$
\left[y^{*}\right](t)=\left[m^{*}\right](t)=\left[m^{* c}\right](t)+\sum_{0 \leq s \leq t}\left\{\Delta m^{*}(s)\right\}^{2} .
$$


It is somewhat instructive to think of the economics of semimartingales and quadratic variation in the following way. We start with $y^{*} \in \mathcal{S} \mathcal{M}$ so that

$$
\mathrm{d} y^{*}=\mathrm{d} \alpha^{*}+\mathrm{d} m^{*} .
$$

If $\alpha^{*} \in \mathcal{F} \mathcal{V}_{\text {loc }}^{c}$ (and so predictable) and if $m^{*} \in \mathcal{M}$ (a martingale) then the predictability of $\alpha^{*}$ means that

$$
\mathrm{E}\left(\mathrm{d} y^{*}(t) \mid \mathcal{F}(t)\right)=\mathrm{d} \alpha^{*}(t)
$$

where $\mathcal{F}(t)$ is the natural filtration of $y^{*}(t)$. Thus the drift can be thought of as

$$
\alpha^{*}(t)=\int_{0}^{t} \mathrm{E}\left(\mathrm{d} y^{*}(u) \mid \mathcal{F}(u)\right)
$$

the integral of the expected instantaneous returns. Additionally, so long as $m^{*} \in \mathcal{M}^{c}$ is locally square integrable then

$$
\begin{aligned}
{\left[y^{*}\right](t) } & =\int_{0}^{t} \operatorname{Var}\left(\mathrm{d} y^{*}(u) \mid \mathcal{F}(u)\right) \\
& =\int_{0}^{t} \operatorname{Var}\left(\mathrm{d} m^{*}(u) \mid \mathcal{F}(u)\right),
\end{aligned}
$$

that is quadratic variation equals the integrated variance of the instantaneous returns. More generally, allowing jumps in the martingale component of prices, so long as $\alpha^{*} \in \mathcal{F} \mathcal{V}_{l o c}^{c}$, we have that

$$
\left[y^{*}\right](t)=\int_{0}^{t} \operatorname{Var}\left(\mathrm{d} m^{* c}(u) \mid \mathcal{F}(u)\right)+\sum_{0 \leq s \leq t}\left\{\Delta m^{*}(s)\right\}^{2} .
$$

Andersen, Bollerslev, Diebold, and Labys (2003) discuss the connection between $\left[y^{*}\right](t)$ and $\operatorname{Var}\left(m^{*}(t) \mid \mathcal{F}(0)\right)$, which is important from a practical forecasting viewpoint.

The above analysis implies that if $y^{*} \in \mathcal{S M}$

$$
\left[y_{M}^{*}\right]_{i}^{[2]}=\sum_{j=1}^{M} y_{j, i}^{2} \stackrel{p}{\rightarrow}\left[y^{*}\right](\hbar i)-\left[y^{*}\right](\hbar(i-1))=\left[y^{*}\right]_{i},
$$

meaning that realised variance consistently estimates daily increments of QV. In turn, so long as $\alpha^{*} \in \mathcal{F} \mathcal{V}_{l o c}^{c}$ then

$$
\left[y_{M}^{*}\right]_{i}^{[2]} \stackrel{p}{\rightarrow}\left[m^{*}\right]_{i}
$$

In addition, if $\alpha^{*} \in \mathcal{F} \mathcal{V}_{l o c}^{c}$ and $m^{*}$ is square integrable, then

$$
\left[y_{M}^{*}\right]_{i}^{[2]} \stackrel{p}{\rightarrow}\left[m^{* c}\right]_{i}+\left[m^{* d}\right]_{i}=\int_{\hbar(i-1)}^{\hbar i} \operatorname{Var}\left(\mathrm{d} m^{* c}(u) \mid \mathcal{F}(u)\right)+\left[m^{* d}\right]_{i},
$$

the integrated variance of the instantaneous returns of the continuous component plus the quadratic variation of the jump part of $m^{*}$. This implies, importantly, that realised variance 
cannot be informative about the separate contributions the diffusion or jump parts of $m^{*}$ make to QV. Instead, in order to break up these two components, we either have to make very specific parametric assumptions about $y^{*}$ (see e.g. Eraker, Johannes, and Polson (2003)) or use alternative aggregate volatility statistics. We follow the latter route in this paper.

\subsection{Stochastic volatility}

To extend the results on quadratic variation we will need some additional smoothness assumptions. We start by stating two, after recalling that $y^{*}=\alpha^{*}+m^{*}$.

(a) $m^{*}$ is a stochastic volatility $(\mathrm{SV})$ process $^{5}$

$$
m^{*}(t)=\int_{0}^{t} \sigma(u) \mathrm{d} w(u)
$$

where $w$ is standard Brownian motion, $\sigma(t)>0$, the spot volatility process, is càdlàg and locally bounded away from zero and the integrated variance process

$$
\sigma^{2 *}(t)=\int_{0}^{t} \sigma^{2}(u) \mathrm{d} u
$$

satisfies $\sigma^{2 *}(t)<\infty$ for all $t<\infty$.

(b) the mean process $\alpha^{*}$ is continuous.

We call semimartingales satisfying (a) and (b) members of the continuous SV semimartingales class (denoted $\mathcal{S} \mathcal{V} \mathcal{S} \mathcal{M}^{c}$ ). Clearly $\mathcal{S} \mathcal{V} \mathcal{S M}^{c} \subset \mathcal{S} \mathcal{M}^{c} \subset \mathcal{S M}$, where $\mathcal{S M}^{c}$ is the class of continuous semimartingales. ${ }^{6}$

Importantly, if $y^{*} \in \mathcal{S} \mathcal{S} \mathcal{M M}^{c}$ then

$$
\left[y^{*}\right](t)=\sigma^{2 *}(t) .
$$

Under the additional assumption that $\sigma$ is continuous $^{7}, \partial\left[y^{*}\right](t) / \partial t=\sigma^{2}(t)$. Finally, for all $y^{*} \in \mathcal{S} \mathcal{V S} \mathcal{M}^{c}$

$$
\left\{y_{M}^{*}\right\}_{i}^{[2]}=\left[y_{M}^{*}\right]_{i}^{[2]}=\sum_{j=1}^{M} y_{j, i}^{2} \stackrel{p}{\rightarrow} \sigma_{i}^{2}, \quad \text { where } \quad \sigma_{i}^{2}=\sigma^{2 *}(\hbar i)-\sigma^{2 *}(\hbar(i-1)) .
$$

\footnotetext{
${ }^{5}$ The literature of SV models is discussed by, for example, Harvey, Ruiz, and Shephard (1994), Taylor (1994), Ghysels, Harvey, and Renault (1996), Shephard (1996) and Kim, Shephard, and Chib (1998).

${ }^{6}$ This construction looks, at first sight, ad hoc. However, this is not true. All local martingales with absolutely continuous quadratic variation can be written in the form of assumption (a). This result, which is due to Doob (1953), is discussed in, for example, Karatzas and Shreve (1991, p. 170-172). If $m^{*} \in \mathcal{M}_{\text {loc }}^{c}$ and $\alpha^{*}$ is predictable then, if we impose a lack of arbitrage opportunity, $\alpha^{*} \in \mathcal{B} \mathcal{V}_{l o c}^{c}$. This condition is discussed in Andersen, Bollerslev, Diebold, and Labys (2003, p. 583). Hence, in the context of financial economics one might argue that assumption (b) follows from (a). From the Dambis-Dubins-Schwartz Theorem we know that the difference between $\mathcal{M}_{l o c}^{c}$ and those given in assumption (a) are the local martingales which have only continuous, not absolutely continuous, quadratic variation. Hence assumptions (a) and (b) are only slightly stronger than saying prices must have continuous sample paths.

${ }^{7}$ More generally, if $D_{-}\{g(x)\}$ denotes the left derivative of a function $g, \lim _{\varepsilon \downarrow 0} \varepsilon^{-1}\{g(x)-g(x-\varepsilon)\}$, then $D_{-}\left\{\left[y^{*}\right](t)\right\}=\sigma^{2}(t-)$. This is relevant when the $\sigma$ process exhibits jumps (e.g. Barndorff-Nielsen and Shephard (2001) and Eraker, Johannes, and Polson (2003)).
} 
We call $\sigma_{i}^{2}$ and $\sqrt{\sigma_{i}^{2}}$ the actual variance and actual volatility, respectively, of the SV component over the $i$-th interval. All these results on SV processes are very well known. They hold irrespective of the relationship between $\alpha^{*}, \sigma$ and $w$.

\subsection{Power variation process}

The quadratic variation process $\left[y^{*}\right]$ was generalised to the power variation process by BarndorffNielsen and Shephard (2003d). Unlike the QV case, power variation is at least initially (see Barndorff-Nielsen and Shephard (2003c) and Barndorff-Nielsen, Graversen, and Shephard (2003) for a discussion of the more general case) defined via an equally spaced discretisation of time. Again we work on the time interval from 0 to $t$, but now we assume we have observations every $\delta>0$ periods of time. Our interest will be in seeing what happens as $\delta \downarrow 0$. This will determine the probability limit of realised power variation $\left\{y_{M}^{*}\right\}_{i}^{[r]}$ defined in (2), which will be given explicitly at the end of this subsection.

The $r$-th order power variation process $(r>0)$ is defined, when it exists, as

$$
\left\{y^{*}\right\}^{[r]}(t)=p-\lim _{\delta \downarrow 0} \delta^{1-r / 2} \sum_{j=1}^{\lfloor t / \delta\rfloor}\left|y_{j}(t)\right|^{r},
$$

where the equally spaced $j$-th return is

$$
y_{j}=y_{j}(t)=y^{*}(j \delta)-y^{*}((j-1) \delta) .
$$

Here, for any real number $a,\lfloor a\rfloor$ denotes the largest integer less than or equal to $a$.

The normalisation $\delta^{1-r / 2}$ is essential in power variation.

1. When $r=2$ the normalisation is one and so disappears.

2. When $r>2$ the normalisation goes off to infinity as $\delta \downarrow 0$.

3. When $r<2$ the normalisation goes to zero as $\delta \downarrow 0$.

The key property of power variation for $\mathcal{S} \mathcal{V} \mathcal{S M}^{c}$ processes is given as follows.

Theorem 1 If $y^{*} \in \mathcal{S} \mathcal{S} \mathcal{M}^{c}$ and additionally $\left(\sigma, \alpha^{*}\right)$ are independent of $w$, then

$$
\left\{y^{*}\right\}^{[r]}(t)=\mu_{r} \int_{0}^{t} \sigma^{r}(s) \mathrm{d} s, \quad \text { where } \quad \mu_{r}=\mathrm{E}|u|^{r}=2^{r / 2} \frac{\Gamma\left(\frac{1}{2}(r+1)\right)}{\Gamma\left(\frac{1}{2}\right)},
$$

for $r>0$ where $u \sim N(0,1)$.

Proof. See Barndorff-Nielsen and Shephard (2003d). 
Remark 1 It is so far unclear to us how substantial is the impact of relaxing the independence condition on $\left(\sigma, \alpha^{*}\right)$ and $w$. Note, no additional assumptions are made on the $\left(\sigma, \alpha^{*}\right)$ process beyond those stated in (a) and (b).

This implies that if $\sigma$ is continuous ${ }^{8},\left(\partial \mu_{r}^{-1}\left\{y^{*}\right\}^{[r]}(t) / \partial t\right)^{1 / r}=\sigma(t)$.

The probability limit of realised power variation follows immediately from the definition of the power variation process. Thus under the conditions of Theorem 1 , as $M \rightarrow \infty$,

$$
\begin{aligned}
\left\{y_{M}^{*}\right\}_{i}^{[r]} & \stackrel{p}{\rightarrow}\left\{y^{*}\right\}^{[r]}(\hbar i)-\left\{y^{*}\right\}^{[r]}(\hbar(i-1)) \\
& =\mu_{r} \int_{\hbar(i-1)}^{\hbar i} \sigma^{r}(s) \mathrm{d} s
\end{aligned}
$$

Barndorff-Nielsen and Shephard (2003d) have extended the above convergence in probability to a distribution theory for $\left\{y^{*}\right\}^{[r]}(t)-\mu_{r} \int_{0}^{t} \sigma^{r}(s) \mathrm{d} s$. This will be discussed in Section 6.4 of this paper. Andreou and Ghysels (2003) have used power variations to test for changes in the level of volatility in financial markets.

\subsection{Bipower variation process}

In the context of multivariate covariation Barndorff-Nielsen and Shephard (2002a) sometimes found it helpful to study cross terms of the type

$$
\delta^{-1} \sum_{j=1}^{\lfloor t / \delta\rfloor-1} y_{j}^{2} y_{j+1}^{2} \stackrel{p}{\rightarrow} \int_{0}^{t} \sigma^{4}(u) \mathrm{d} u,
$$

where we recall that $y_{j}=y^{*}(j \delta)-y^{*}((j-1) \delta)$. Here we provide an extension of this which will allow us to understand realised bipower variation, the key innovation in this paper. First we define the bipower variation (BPV) process as

$$
\left\{y^{*}\right\}^{[r, s]}(t)=p-\lim _{\delta \downarrow 0} \delta^{1-(r+s) / 2} \sum_{j=1}^{\lfloor t / \delta\rfloor-1}\left|y_{j}\right|^{r}\left|y_{j+1}\right|^{s}, \quad r, s \geq 0,
$$

provided this exists. We will define a more general $k$-th order power variation process in Section 6.2. Clearly the value of $r+s$ is crucial here, as it determines the $\delta$ normalisation. Importantly

$$
\left\{y^{*}\right\}^{[r, 0]}(t)=\left\{y^{*}\right\}^{[0, r]}(t)=\left\{y^{*}\right\}^{[r]}(t) \text {. }
$$

Hence special cases of the BPV process include the power variation process and so, obviously, QV.

If $y^{*} \in \mathcal{S} \mathcal{S} \mathcal{M}^{c}$ and additionally $\left(\sigma, \alpha^{*}\right)$ are independent of $w$, then we would expect that for $r+s>0$

$$
{ }^{8} \text { Otherwise }\left(D_{-}\left[\mu_{r}^{-1}\left\{y^{*}\right\}^{[r]}(t)\right]\right)^{1 / r} \text { is } \sigma(t-) \text {. }
$$




$$
\left\{y^{*}\right\}^{[r, s]}(t)=\mu_{r} \mu_{s} \int_{0}^{t} \sigma^{r+s}(u) \mathrm{d} u, \quad \text { where } \quad \mu_{r}=2^{r / 2} \frac{\Gamma\left(\frac{1}{2}(r+1)\right)}{\Gamma\left(\frac{1}{2}\right)} .
$$

We will discuss a proof of this general result elsewhere. Again the importance of the assumed independence assumption is unclear. Overall, this result provides a second way of accessing integrated power volatility, extending previous work on realised power variation. For now we prove a special case.

Theorem 2 Suppose $y^{*} \in \mathcal{S} \mathcal{V} \mathcal{S M}^{c}$ and additionally $\sigma$ is independent of $w$ and $\alpha^{*}=0$. Then, for any $r>0$, we have

$$
\left\{y^{*}\right\}^{[r, r]}(t)=\mu_{r}^{2} \int_{0}^{t} \sigma^{2 r}(s) \mathrm{d} s .
$$

Proof. It is convenient to introduce the notation

$$
\left\{y_{\delta}^{*}\right\}^{[r, r]}(t)=\delta^{1-r} \sum_{j=1}^{\lfloor t / \delta\rfloor-1}\left|y_{j}\right|^{r}\left|y_{j+1}\right|^{r}
$$

Here, and elsewhere, we use $\delta$ rather than $M$ as lower index. The value of the bipower process $\left\{y^{*}\right\}^{[r, r]}$ at time $t$ is the probability limit, as $\delta \downarrow 0$, of $\left\{y_{\delta}^{*}\right\}^{[r, r]}(t)$. In determining this limit it causes no loss of generality to assume that $t / \delta$ is an integer $M$, say.

Let $\sigma_{j}>0$ be defined by

$$
\sigma_{j}^{2}=\sigma^{2 *}(j \delta)-\sigma^{2 *}((j-1) \delta)
$$

We then have

$$
\left\{y_{\delta}^{*}\right\}^{[r, r]}(t) \stackrel{l a w}{=} \delta^{1-r} \sum_{j=1}^{M-1} \sigma_{j}^{r} \sigma_{j+1}^{r}\left|u_{j}\right|^{r}\left|u_{j+1}\right|^{r}
$$

where the $u_{j}$ are i.i.d. standard normal. This means that

$$
\left\{y_{\delta}^{*}\right\}^{[r, r]}(t)-\mu_{r}^{2} \delta^{1-r} \sum_{j=1}^{M} \sigma_{j}^{r} \sigma_{j+1}^{r} \stackrel{l a w}{=} \delta^{1-r} \sum_{j=1}^{M-1} \sigma_{j}^{r} \sigma_{j+1}^{r}\left(\left|u_{j}\right|\left|u_{j+1}\right|-\mu_{r}^{2}\right) .
$$

Hence in our proof we need to show three things:

1. As $\delta \downarrow 0$

$$
R=\delta^{1-r} \sum_{j=1}^{M-1} \sigma_{j}^{r} \sigma_{j+1}^{r}\left(\left|u_{j}\right|\left|u_{j+1}\right|-\mu_{r}^{2}\right) \stackrel{p}{\rightarrow} 0
$$

2. As $\delta \downarrow 0$

$$
\delta^{1-r}\left\{\sum_{j=1}^{M-1} \sigma_{j}^{r} \sigma_{j+1}^{r}-\sum_{j=1}^{M} \sigma_{j}^{2 r}\right\} \stackrel{p}{\rightarrow} 0
$$

3. As $\delta \downarrow 0$

$$
\delta^{1-r} \sum_{j=1}^{M} \sigma_{j}^{2 r} \stackrel{p}{\rightarrow} \sigma^{2 r *}(t)
$$


Point 3 is straightforward using Riemann integrability of $\sigma(t)$ (e.g. Barndorff-Nielsen and Shephard (2003d)).

We now take up part 1 . To prove (11), let $R^{\prime}$ be an independent copy of $R$ and note that $R+R^{\prime}$ can be rearranged as the sum of two sums each of the form

$$
S=\delta^{1-r} \sum_{j=1}^{M-1} \sigma_{j}^{r} \sigma_{j+1}^{r}\left(w_{j}-\mu_{r}^{2}\right)
$$

where the $w_{j}$ are i.i.d. and distributed as $|u|\left|u^{\prime}\right|$ with $u$ and $u^{\prime}$ independent standard normal. Barndorff-Nielsen and Shephard (2003c, Corollary 4.3) applies to such a sum, as we indicate below, yielding $S \stackrel{p}{\rightarrow} 0$. Therefore $R+R^{\prime} \stackrel{p}{\rightarrow} 0$ and as $R$ and $R^{\prime}$ are independent the same is true of $R$.

To see that $S \stackrel{p}{\rightarrow} 0$ let

$$
c_{M j}=\delta^{1-r} \sum_{j=1}^{M-1} \sigma_{j}^{r} \sigma_{j+1}^{r}
$$

and

$$
x=w^{2}-\mu_{2 r}
$$

where $w$ is standard normal. Then, as is simple to verify, the following three conditions are satisfied

$$
\begin{gathered}
c_{M}=\max _{1 \leq j<M} c_{M j} \rightarrow 0 \\
M P\left\{|x| \geq c_{M}^{-1} \varepsilon\right\} \rightarrow 0 \\
\sup _{M} \sum_{j=1}^{M-1} c_{M j}<\infty
\end{gathered}
$$

and thus Barndorff-Nielsen and Shephard (2003c, Corollary 4.3) applies.

It only remains to prove part 2 with (12). In fact we have the stronger result that

$$
\delta^{1-r}\left\{\sum_{j=1}^{M-1} \sigma_{j}^{r} \sigma_{j+1}^{r}-\sum_{j=1}^{M} \sigma_{j}^{2 r}\right\}=O(\delta) .
$$


To establish this rewrite the difference as follows

$$
\begin{aligned}
\sum_{j=1}^{M-1} \sigma_{j}^{r} \sigma_{j+1}^{r}-\sum_{j=1}^{M} \sigma_{j}^{2 r} & =\sum_{j=1}^{M-1}\left(\sigma_{j}^{r} \sigma_{j+1}^{r}-\sigma_{j}^{2 r}\right)-\sigma_{M}^{2 r} \\
& =\sum_{j=1}^{M-1} \sigma_{j}^{r}\left(\sigma_{j+1}^{r}-\sigma_{j}^{r}\right)-\sigma_{M}^{2 r} \\
& =\sum_{j=1}^{M-1} \frac{\sigma_{j}^{r}}{\sigma_{j+1}^{r}+\sigma_{j}^{r}}\left(\sigma_{j+1}^{2 r}-\sigma_{j}^{2 r}\right)-\sigma_{M}^{2 r} \\
& =\frac{1}{2} \sum_{j=1}^{M-1}\left(\frac{2 \sigma_{j}^{r}}{\sigma_{j+1}^{r}+\sigma_{j}^{r}}-1\right)\left(\sigma_{j+1}^{2 r}-\sigma_{j}^{2 r}\right)-\frac{1}{2}\left(\sigma_{1}^{2 r}+\sigma_{M}^{2 r}\right) \\
& =-\frac{1}{2} \sum_{j=1}^{M-1} \frac{\sigma_{j+1}^{r}-\sigma_{j}^{r}}{\sigma_{j+1}^{r}+\sigma_{j}^{r}}\left(\sigma_{j+1}^{2 r}-\sigma_{j}^{2 r}\right)-\frac{1}{2}\left(\sigma_{1}^{2 r}+\sigma_{M}^{2 r}\right) \\
& =-\frac{1}{2} \sum_{j=1}^{M-1} \frac{\left(\sigma_{j+1}^{2 r}-\sigma_{j}^{2 r}\right)^{2}}{\left(\sigma_{j+1}^{r}+\sigma_{j}^{r}\right)^{2}}-\frac{1}{2}\left(\sigma_{1}^{2 r}+\sigma_{M}^{2 r}\right) .
\end{aligned}
$$

Further, letting $\psi_{j}=\delta^{-1 / 2} \sigma_{j}$ we find

$$
\delta^{1-r}\left\{\sum_{j=1}^{M-1} \sigma_{j}^{r} \sigma_{j+1}^{r}-\sum_{j=1}^{M} \sigma_{j}^{2 r}\right\}=-\frac{1}{2} \delta\left\{\sum_{j=1}^{M-1} \frac{\left(\psi_{j+1}^{2 r}-\psi_{j}^{2 r}\right)^{2}}{\left(\psi_{j+1}^{r}+\psi_{j}^{r}\right)^{2}}+\psi_{1}^{2 r}+\psi_{M}^{2 r}\right\} .
$$

By condition (a) we have, in particular, that

$$
0<\inf _{0 \leq s \leq t} \sigma(s) \leq \inf \psi_{j} \leq \sup \psi_{j} \leq \sup _{0 \leq s \leq t} \sigma(s)<\infty
$$

uniformly in $\delta$. From this it is immediate that $\psi_{1}^{2 r}+\psi_{M}^{2 r}$ is uniformly bounded from above. Furthermore,

$$
\sum_{j=1}^{M-1} \frac{\left(\psi_{j+1}^{2 r}-\psi_{j}^{2 r}\right)^{2}}{\left(\psi_{j+1}^{r}+\psi_{j}^{r}\right)^{2}} \leq \frac{1}{4} \frac{1}{\inf _{0 \leq s \leq t} \sigma^{2 r}(s)} \sum_{j=1}^{M-1}\left(\psi_{j+1}^{2 r}-\psi_{j}^{2 r}\right)^{2},
$$

where $\inf _{0 \leq s \leq t} \sigma^{2 r}(s)>0$ and, by the càdlàg property of $\sigma$,

$$
\sum_{j=1}^{M-1}\left(\psi_{j+1}^{2 r}-\psi_{j}^{2 r}\right)^{2} \stackrel{p}{\rightarrow}\left[\sigma^{2 r}\right](t)
$$

which implies, together with (14), result (13) and hence (10).

Following the initial draft of this paper, Barndorff-Nielsen and Shephard (2003a) have shown that the result is not effected by replacing the $\alpha^{*}=0$ condition by a much weaker smoothness assumption on $\alpha^{*}$. 
The above result implies the realised bipower variation

$$
\mu_{r}^{-1} \mu_{s}^{-1}\left\{y_{M}^{*}\right\}_{i}^{[r, s]} \stackrel{p}{\rightarrow} \int_{\hbar(i-1)}^{\hbar i} \sigma^{r+s}(u) \mathrm{d} u .
$$

Clearly

$$
\mu_{r}^{-1} \mu_{2-r}^{-1}\left\{y_{M}^{*}\right\}_{i}^{[r, 2-r]} \stackrel{p}{\rightarrow} \int_{\hbar(i-1)}^{\hbar i} \sigma^{2}(u) \mathrm{d} u, \quad r \in(0,2),
$$

a consistent estimator of integrated variance and so gives the same result as the realised variance. Further, we have the simple result in the $\mathcal{S} \mathcal{S} \mathcal{S M}^{c}$ case that

$$
\left[y_{M}^{*}\right]_{i}^{[2]}-\mu_{r}^{-1} \mu_{2-r}^{-1}\left\{y_{M}^{*}\right\}_{i}^{[r, 2-r]}(t) \stackrel{p}{\rightarrow} 0, \quad r \in(0,2) .
$$

We will see in the next Section that when there are jumps this term will not have a zero probability limit, but rather will converge to a positive, finite quantity. It will become clear that this difference can be used to test for the presence of jumps.

We now introduce an additional regularity condition.

Condition $\left(\mathbf{V}^{\prime}\right)$. The volatility process $\sigma$ has the property

$$
\lim _{\delta \downarrow 0} \delta^{1 / 2} \sum_{j=1}^{M}\left|\sigma^{r}\left(\eta_{j}\right)-\sigma^{r}\left(\xi_{j}\right)\right|=0,
$$

for some $r>0$ (equivalently for every $r>0$ ) and for any $\xi_{j}=\xi_{j}(\delta)$ and $\eta_{j}=\eta_{j}(\delta)$ such that

$$
0 \leq \xi_{1} \leq \eta_{1} \leq \delta \leq \xi_{2} \leq \eta_{2} \leq 2 \delta \leq \cdots \leq \xi_{M} \leq \eta_{M} \leq t .
$$

Using this condition we may strengthen Theorem 2 to the following statement.

Theorem 3 Let the situation be as in Theorem 2 and suppose moreover that $\sigma$ satisfies condition $\left(V^{\prime}\right)$. Then

$$
\delta^{1-r} \sum_{j=1}^{\lfloor t / \delta\rfloor-1}\left|y_{j}\right|^{r}\left|y_{j+1}\right|^{r}=\mu_{r}^{2} \int_{0}^{t} \sigma^{2 r}(s) \mathrm{d} s+o_{p}\left(\delta^{1 / 2}\right) .
$$

Proof By the proof of Theorem 2, particularly the conclusion (13), it suffices to note that when conditions (a) and $\left(\mathbf{V}^{\prime}\right)$ hold, then together they imply that

$$
\delta^{1-r} \sum_{j=1}^{M} \sigma_{j}^{2 r}-\sigma^{2 r *}(t)=o_{p}\left(\delta^{1 / 2}\right) .
$$

This latter relation is in fact the content of Barndorff-Nielsen and Shephard (2003c, Lemma 2).

The result (15) given in Theorem 3 is essential in deriving distributional limit results for realised power variation. See Section 6.4 below. Barndorff-Nielsen and Shephard (2003a) have recently shown that in the case where $r=1$ condition $\left(\mathbf{V}^{\prime}\right)$ can be dispensed with. 


\section{SV process plus large but rare jumps}

\subsection{Rare jumps and quadratic variation}

So far we have seen that in $\mathcal{S} \mathcal{V} \mathcal{S M}^{c}$ models realised variance, realised power variation and realised bipower variation converge to integrals of powers of spot volatility. When we use special cases of realised bipower variation, then the probability limit is the same as that achieved by realised variance. In this section we will see how this result changes when we introduce jumps.

Consider the log-price process

$$
y^{*}(t)=y^{(1) *}(t)+y^{(2) *}(t),
$$

with $y^{(1) *} \in \mathcal{S} \mathcal{V} \mathcal{S} \mathcal{M}^{c}$ and

$$
y^{(2) *}(t)=\sum_{i=1}^{N(t)} c_{i},
$$

where $N$ is a finite activity, simple counting process ${ }^{9}$ such that $N(t)<\infty$ (for all $t>0$ ) and $\left\{c_{i}\right\}$ is a collection of non-zero random variables. In the special case where $N$ is a homogeneous Poisson process and $\left\{c_{i}\right\}$ are i.i.d. from some distribution $D$ then $y^{(2) *}$ is a compound Poisson process (written $\mathcal{C P} \mathcal{P}$ ). Such jump process models have often been used as components of price processes (e.g. Merton (1976), Andersen, Benzoni, and Lund (2002), Johannes, Polson, and Stroud (2002) and Chernov, Gallant, Ghysels, and Tauchen (2003)).

The QV for this jump plus $\mathcal{S} \mathcal{V} \mathcal{S} \mathcal{M}^{c}$ process is well known (see e.g. the discussion in Andersen, Bollerslev, Diebold, and Labys (2001)) and is reported below in the following proposition.

Proposition 1 Suppose $y^{(1) *} \in \mathcal{S} \mathcal{V} \mathcal{S} \mathcal{M}^{c}$ and $y^{(2) *}$ is given in (17), then

$$
\begin{aligned}
{\left[y^{*}\right](t) } & =\sigma^{2 *}(t)+\sum_{i=1}^{N(t)} c_{i}^{2} \\
& =\left[y^{(1) *}\right]^{[2]}(t)+\left[y^{(2) *}\right]^{[2]}(t) .
\end{aligned}
$$

Proof. Trivial from (6) and (8).

\subsection{Rare jumps and power variation}

We can generalise the above discussion to deal with power variation. This captures the theoretical effect we are concerned with in this paper.

\footnotetext{
${ }^{9} \mathrm{~A}$ finite activity counting process is a simple counting process which, with probability one, has a finite number of events happening in a fixed interval of time. This rules out infinite activity Lévy processes such as the normal inverse Gaussian process which have an infinite number of jumps in finite intervals of time. These processes will be discussed in Section 6 .
} 
Theorem 4 Let $y^{*}=y^{(1) *}+y^{(2) *}$ with $y^{(1) *}$ and $y^{(2) *}$ being independent. Suppose $y^{(1) *} \in$ $\mathcal{S} \mathcal{S} \mathcal{M}^{c}$, that is $y^{*}=\alpha^{*}+m^{*}$, and additionally that $\left(\sigma, \alpha^{*}\right)$ are independent of $w$ and $y^{(2) *}$ is given in (17). Then

$$
\mu_{r}^{-1}\left\{y^{*}\right\}^{[r]}(t)= \begin{cases}\int_{0}^{t} \sigma^{r}(u) \mathrm{d} u, & r \in(0,2) \\ {\left[y^{*}\right](t),} & r=2 \\ \infty, & r>2\end{cases}
$$

Remark 2 The same setting has been independently studied recently by Woerner (2002), who reaches the same conclusion in a general semimartingale framework using a different technique. See also Lépingle (1976) for an earlier investigation.

Proof. It is clear that as $\delta \downarrow 0$, then generally

$$
\sum_{j=1}^{\lfloor t / \delta\rfloor}\left|y_{j}^{(2)}(t)\right|^{r} \stackrel{p}{\rightarrow} \sum_{i=1}^{N(t)}\left|c_{i}\right|^{r}
$$

where

$$
y_{j}^{(2)}(t)=y^{(2) *}(j \delta)-y^{(2) *}((j-1) \delta) .
$$

It follows from this that when we also normalise the sum, as $\delta \downarrow 0$

$$
\delta^{\beta} \sum_{j=1}^{\lfloor t / \delta\rfloor}\left|y_{j}^{(2)}(t)\right|^{r} \stackrel{p}{\rightarrow} \begin{cases}0 & \text { for } \beta>0, r \in(0,2) \\ {\left[y^{(2) *}\right](t)} & \text { for } \beta=0, r=2 \\ \infty & \text { for } \beta<0, r>2 .\end{cases}
$$

Hence the power variation of $y^{(2) *}$ is either zero, the quadratic variation or infinity, depending upon the value of $r$. Furthermore

$$
\sum_{j=1}^{\lfloor t / \delta\rfloor}\left|y_{j}(t)\right|^{r}=\sum_{j=1}^{\lfloor t / \delta\rfloor}\left|y_{j}^{(1)}(t)\right|^{r}+O_{p}(N(t)),
$$

as there are only $N(t)$ contributions to $y^{(2) *}$. As a result we have that

$$
\mu_{r}^{-1} \delta^{\beta} \sum_{j=1}^{\lfloor t / \delta\rfloor}\left|y_{j}(t)\right|^{r} \stackrel{p}{\rightarrow} \begin{cases}\int_{0}^{t} \sigma^{r}(u) \mathrm{d} u, & \text { for } \beta=1-r / 2, r \in(0,2) \\ {\left[y^{*}\right](t),} & \text { for } \beta=0, r=2 \\ \infty, & \text { for } \beta<0, r>2 .\end{cases}
$$

This delivers the required result by definition of $\left\{y^{*}\right\}^{[r]}(t)$.

Theorem 4 implies that:

1. When $r \in(0,2)$ the probability limit of realised power variation is unaffected by the presence of the jumps. 
2. When $r>2$ the probability limit is determined by the jump component and so scaling means this goes off to infinity.

3. When $r=2$ both components contribute.

The result is not very sensitive for it would hold for any jump process as long as there are a finite number of jumps in any finite period of time. In particular, then, the jumps can be serially dependent, or dependent on the filtration generated by $w$ and $\sigma$. The above implies that when $r<2$ and $\sigma$ is continuous ${ }^{10}$ then $\sigma(t)=\left(\mu_{r}^{-1} \frac{\partial\left\{y^{*}\right\}^{[r]}(t)}{\partial t}\right)^{1 / r}$.

\subsection{Rare jumps and bipower variation}

The logic of the proof of Theorem 4 carries over to the bipower variation process. In particular we deliver the stimulating result on bipower given in the following theorem ${ }^{11}$. The only part of the result we will use in practice is the $\max (r, s)<2$ case.

Theorem 5 Let the setting be as in Theorem 4. Then for $r>0$ and $s>0$

$$
\mu_{r}^{-1} \mu_{s}^{-1}\left\{y^{*}\right\}^{[r, s]}(t)= \begin{cases}\int_{0}^{t} \sigma^{r+s}(u) \mathrm{d} u, & \max (r, s)<2, \\ x^{*}(t) & \max (r, s)=2, \\ \infty, & \max (r, s)>2,\end{cases}
$$

where $x^{*}(t)$ is some stochastic process.

Proof. Defining

$$
d_{j, j+1}= \begin{cases}1, & y_{j}^{(2)}=y_{j+1}^{(2)}=0 \\ 1, & y_{j}^{(2)} \neq 0, y_{j+1}^{(2)} \neq 0 \\ 0, & \text { elsewhere }\end{cases}
$$

we have an indicator which is one either if there are no jumps or the jumps are contiguous. Then

$$
\begin{aligned}
& \delta^{1-(r+s) / 2} \sum_{j=1}^{\lfloor t / \delta\rfloor-1}\left|y_{j}\right|^{r}\left|y_{j+1}\right|^{s} \\
= & \delta^{1-(r+s) / 2} \sum_{j=1}^{\lfloor t / \delta\rfloor-1}\left|y_{j}\right|^{r}\left|y_{j+1}\right|^{s} d_{j, j+1}+\delta^{1-(r+s) / 2} \sum_{j=1}^{\lfloor t / \delta\rfloor-1}\left|y_{j}\right|^{r}\left|y_{j+1}\right|^{s}\left(1-d_{j, j+1}\right) .
\end{aligned}
$$

The impact of the jumps on the first term in (20) of any contiguous jumps goes to zero as $\delta \downarrow 0$ so long as $r+s<2$, for the jumps contribute a finite number of terms to the sum and so

$$
\delta^{1-(r+s) / 2} \sum_{j=1}^{\lfloor t / \delta\rfloor-1}\left|y_{j}\right|^{r}\left|y_{j+1}\right|^{s} d_{j, j+1}-\delta^{1-(r+s) / 2} \sum_{j=1}^{\lfloor t / \delta\rfloor-1}\left|y_{j}^{(1)}\right|^{r}\left|y_{j+1}^{(1)}\right|^{s} \stackrel{p}{\rightarrow} 0,
$$

\footnotetext{
${ }^{10}$ Otherwise when $r<2$ then $\left(D_{-}\left[\mu_{r}^{-1}\left\{y^{*}\right\}^{[r]}(t)\right]\right)^{1 / r}$ is $\sigma(t-)$.

${ }^{11}$ We thank Xin Huang from Duke University for pointing out an error in an earlier version of this result and for deriving the $\max (r, s)=2$ case.
} 
as the contribution from the contiguous jumps is killed by the scaling $\delta^{1-(r+s) / 2} \downarrow 0$ as $\delta \downarrow 0$. The condition on $r$ and $s$ can be relaxed to $\max (r, s)<2$ as long as the probability of two contiguous jumps goes to zero at rate $\delta$, which is true for simple counting processes. Of course, under the conditions of the Theorem

$$
\delta^{1-(r+s) / 2} \sum_{j=1}^{\lfloor t / \delta\rfloor-1}\left|y_{j}^{(1)}\right|^{r}\left|y_{j+1}^{(1)}\right|^{s} \stackrel{p}{\rightarrow} \int_{0}^{t} \sigma^{r+s}(u) \mathrm{d} u
$$

for all $r$ and $s$.

We now work with

$$
\sum_{j=1}^{\lfloor t / \delta\rfloor-1}\left|y_{j}\right|^{r}\left|y_{j+1}\right|^{s}\left(1-d_{j, j+1}\right)=O(N(t))
$$

as the terms in the sum are zero unless there is a jump and there are $N(t)$ jumps. The focus of attention is on

$$
\begin{aligned}
& \delta^{1-(r+s) / 2} \sum_{j=1}^{\lfloor t / \delta\rfloor-1}\left|y_{j}\right|^{r}\left|y_{j+1}\right|^{s}\left(1-d_{j, j+1}\right) \\
\leq & \delta^{1-(r+s) / 2} \sum_{j=1}^{\lfloor t / \delta\rfloor-1}\left|y_{j}^{(1)}\right|^{r}\left|y_{j+1}^{(1)}+y_{j+1}^{(2)}\right|^{s}\left(1-d_{j, j+1}\right) \\
& +\delta^{1-(r+s) / 2} \sum_{j=1}^{\lfloor t / \delta\rfloor-1}\left|y_{j}^{(1)}+y_{j}^{(2)}\right|^{r}\left|y_{j+1}^{(1)}\right|^{s}\left(1-d_{j, j+1}\right) \\
= & \delta^{1-s / 2} \sum_{j=1}^{\lfloor t / \delta\rfloor-1}\left|\frac{y_{j}^{(1)}}{\sqrt{\delta}}\right|^{r}\left|y_{j+1}^{(1)}+y_{j+1}^{(2)}\right|^{s}\left(1-d_{j, j+1}\right) \\
& +\delta^{1-r / 2} \sum_{j=1}^{\lfloor t / \delta\rfloor-1}\left|y_{j}^{(1)}+y_{j}^{(2)}\right|^{r}\left|\frac{y_{j+1}^{(1)}}{\sqrt{\delta}}\right|^{s}\left(1-d_{j, j+1}\right) .
\end{aligned}
$$

So long as $s<2$ then

$$
\delta^{1-s / 2} \sum_{j=1}^{\lfloor t / \delta\rfloor-1}\left|\frac{y_{j}^{(1)}}{\sqrt{\delta}}\right|^{r}\left|y_{j+1}^{(1)}+y_{j+1}^{(2)}\right|^{s}\left(1-d_{j, j+1}\right) \stackrel{p}{\rightarrow} 0,
$$

while likewise if $r<2$ then

$$
\delta^{1-r / 2} \sum_{j=1}^{\lfloor t / \delta\rfloor-1}\left|y_{j}^{(1)}+y_{j}^{(2)}\right|^{r}\left|\frac{y_{j+1}^{(1)}}{\sqrt{\delta}}\right|^{s}\left(1-d_{j, j+1}\right) \stackrel{p}{\rightarrow} 0 .
$$

This delivers the first result in (19), while if $\max (r, s)>2$ both of these terms explode which is clearly enough to guarantee the third result in (19).

The only issue which remains is when $\max (r, s)=2$. In that case

$$
\delta^{1-(r+s) / 2} \sum_{j=1}^{\lfloor t / \delta\rfloor-1}\left|y_{j}\right|^{r}\left|y_{j+1}\right|^{s}\left(1-d_{j, j+1}\right)
$$


has a stochastic limit which combines the jumps and the volatility process.

Remark 3 This result has a special case, of a great deal of applied interest. When $r \in(0,2)$ then

$$
\mu_{r}^{-1} \mu_{2-r}^{-1}\left\{y^{*}\right\}^{[r, 2-r]}(t)=\int_{0}^{t} \sigma^{2}(u) \mathrm{d} u,
$$

integrated variance. Hence the integrated variance can be consistently estimated in the presence of rare but large jumps. It also immediately implies that

$$
\left[y^{*}\right]^{[2]}(t)-\mu_{r}^{-1} \mu_{2-r}^{-1}\left\{y^{*}\right\}^{[r, 2-r]}(t)=\sum_{i=1}^{N(t)} c_{i}^{2},
$$

the jump contribution to the QV. We believe this is the first analysis which has been able to feasibly decompose the quadratic variation into the contributions from the continuous and jump components of the log-price. Trivially, we also have that

$$
\mu_{r}^{-1} \mu_{2-r}^{-1}\left\{y^{*}\right\}^{[r, 2-r]}(t)=\int_{0}^{t} \operatorname{Var}\left(\mathrm{d} m^{* c}(u) \mid \mathcal{F}(u)\right),
$$

even when there are jumps in $m^{*}$ and so in $y^{*}$.

Remark 4 The above results imply that realised bipower variation has the feature that when $r \in(0,2)$, then

$$
\mu_{r}^{-1} \mu_{2-r}^{-1}\left\{y_{M}^{*}\right\}_{i}^{[r, 2-r]} \stackrel{p}{\rightarrow} \int_{\hbar(i-1)}^{\hbar i} \sigma^{2}(u) \mathrm{d} u=\int_{\hbar(i-1)}^{\hbar i} \operatorname{Var}\left(\mathrm{d} m^{* c}(u) \mid \mathcal{F}(u)\right),
$$

while

$$
\left\{y_{M}^{*}\right\}_{i}^{[2]}-\mu_{r}^{-1} \mu_{2-r}^{-1}\left\{y_{M}^{*}\right\}_{i}^{[r, 2-r]} \stackrel{p}{\rightarrow} \sum_{j=N(\hbar(i-1))+1}^{N(\hbar i)} c_{j}^{2} .
$$

Thus the use of these statistics can, in theory, separately identify the risk associated with the diffuse element of prices and the jumps.

\section{Some simulations of SV plus large rare jumps}

\subsection{Basic simulation}

In this Section we will illustrate some of these results on realised bipower variation by simulating an SV plus jump process. Throughout we focus on using two special cases of the results given in (21) and (22). In particular we will study

$$
\mu_{1}^{-2}\left\{y_{M}^{*}\right\}_{i}^{[1,1]}=\mu_{1}^{-2} \sum_{j=1}^{M-1}\left|y_{j, i}\right|\left|y_{j+1, i}\right| \stackrel{p}{\rightarrow} \int_{\hbar(i-1)}^{\hbar i} \sigma^{2}(u) \mathrm{d} u
$$


and that

$$
\left[y_{M}^{*}\right]_{i}^{[2]}-\mu_{1}^{-2}\left\{y_{M}^{*}\right\}_{i}^{[1,1]}=\sum_{j=1}^{M} y_{j, i}^{2}-\mu_{1}^{-2} \sum_{j=1}^{M-1}\left|y_{j, i}\right|\left|y_{j+1, i}\right| \stackrel{p}{\rightarrow} \sum_{j=N(\hbar(i-1))+1}^{N(\hbar i)} c_{j}^{2} .
$$

Our aim is to see if these convergence results are of any relevance in finite samples.

We start with a discussion of the spot volatility process. We use a Feller or Cox, Ingersoll, and Ross (1985) square root process for $\sigma^{2}$

$$
\mathrm{d} \sigma^{2}(t)=-\lambda\left\{\sigma^{2}(t)-\xi\right\} \mathrm{d} t+\omega \sigma(t) \mathrm{d} b(\lambda t), \quad \xi \geq \omega^{2} / 2, \quad \lambda>0,
$$

where $b$ is a standard Brownian motion process. The square root process has a marginal distribution

$$
\sigma^{2}(t) \sim \Gamma\left(2 \omega^{-2} \xi, 2 \omega^{-2}\right)=\Gamma(\nu, a), \quad \nu \geq 1,
$$

with a mean of $\xi=\nu / a$, a variance of $\omega^{2}=\nu / a^{2}$ and

$$
\operatorname{Cor}\left\{\sigma^{2}(t), \sigma^{2}(t+s)\right\}=\exp (-\lambda|s|) \text {. }
$$

In the context of SV models this is often called the Heston (1993) model. We take $\alpha^{*}=0$ and so

$$
y^{*}(t)=\int_{0}^{t} \sigma(u) \mathrm{d} w(u)+\sum_{i=1}^{N(t)} c_{i} .
$$

We rule out leverage (e.g. Black (1976) and Nelson (1991)) by assuming Cor $\{b(\lambda t), w(t)\}=0$. We take $\hbar=1, \lambda=0.01, \nu=0.1$ and $a=0.2$. We randomly scatter 10 jumps in a time interval of 50 days, while the jumps are $N I D(0,0.64 \nu / a)$. The latter means that when there is a jump, the jump has the same variance as that expected over $64 \%$ of a day period of trading when there is no jump. Thus these are large but quite rare jumps.

We report results based on $M=12, M=72$ and $M=288$, which are typical practical values for these types of methods. The first row of Figure 2 corresponds to $M=12$, the second has $M=72$ and the third $M=288$. Figures 2(a), (c) and (e) show the sample path of the $y^{*}$ and $N$ processes recorded at the resolution of $M$. Notice we have not used a standard time series plot here; rather we plot the processes using dots so that it is possible to see the jumps. As $M$ increases the sample path of the discretised process reveals the jump in the process.

Figures 2(b), (d) and (f) show the sample paths of the daily realised variances, while we also draw the corresponding daily increments in the quadratic variation, which is obviously the sum of the integrated variance and the sums of squared jumps. We see that the RV becomes more accurate with $M$, as we expect from theory. However, we can also see that it is quite an inaccurate estimator. 

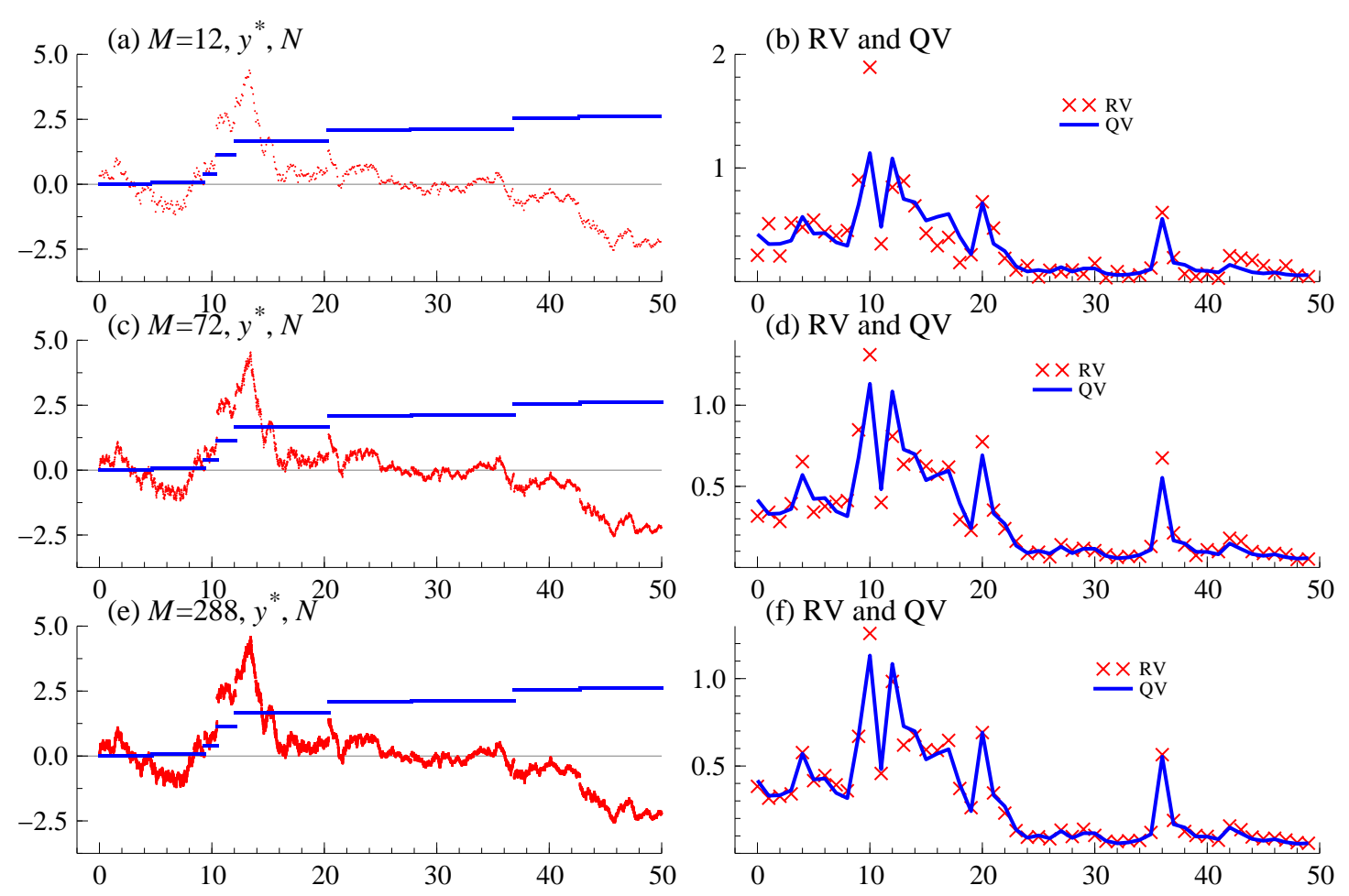

Figure 2: Simulation from a jump plus diffusion based $S V$ model using a square root variance process. (a), (c) and (e) show $y^{*}(t)$ and $0.5 N(t)$. The latter shows the position of the jumps. (b), (d) and (f) show $\sigma_{i}^{2}$ and $\left[y_{M}^{*}\right]_{i}^{[2]}$

Figures 3(a), (c) and (e) show the more innovative results. It displays our estimator of the integrated variance, using the theory of realised bipower variation. In particular, as mentioned above, we are using $r=s=1$ in this work. This is contrasted with the actual increment of the integrated variance. We can see that when $M=12$ this is a poor estimator and is, in particular, influenced by the large jumps which appear in the sample. However for moderate $M$ the statistic seems to be quite informative, while when $M=288$ the estimator is reasonably accurate. Figures 3(b), (d) and (f) show the difference between the realised variance and the realised bipower variation. The theory suggests this is a consistent estimator of the quadratic variation of the jump component. For small $M$ it is very inaccurate, but for moderate and large $M$ the estimator works rather well.

We back up these results by repeating the above analysis but using 5,000 days, having jumps of the same size as before but now on average every 20 days. We then focus on the distribution of the estimation errors. In particular we look in detail at

$$
\left[y^{*}\right]_{i}-\left[y_{M}^{*}\right]_{i}^{[2]},
$$


(a) $\mathrm{M}=12$, Esti \& true int var

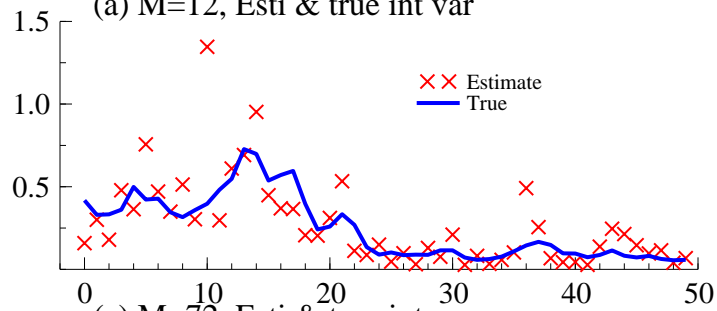

(c) $M=72$, Esti \& true int var

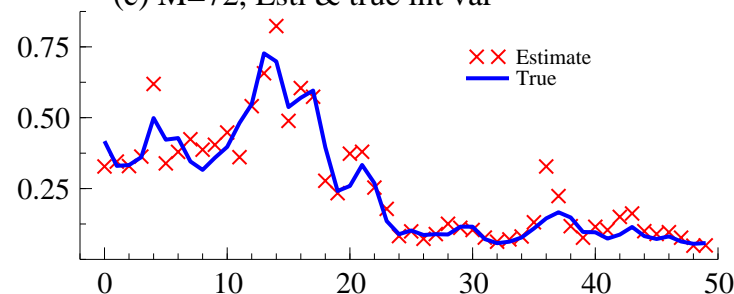

(e) $M=288$, Esti \& true int var
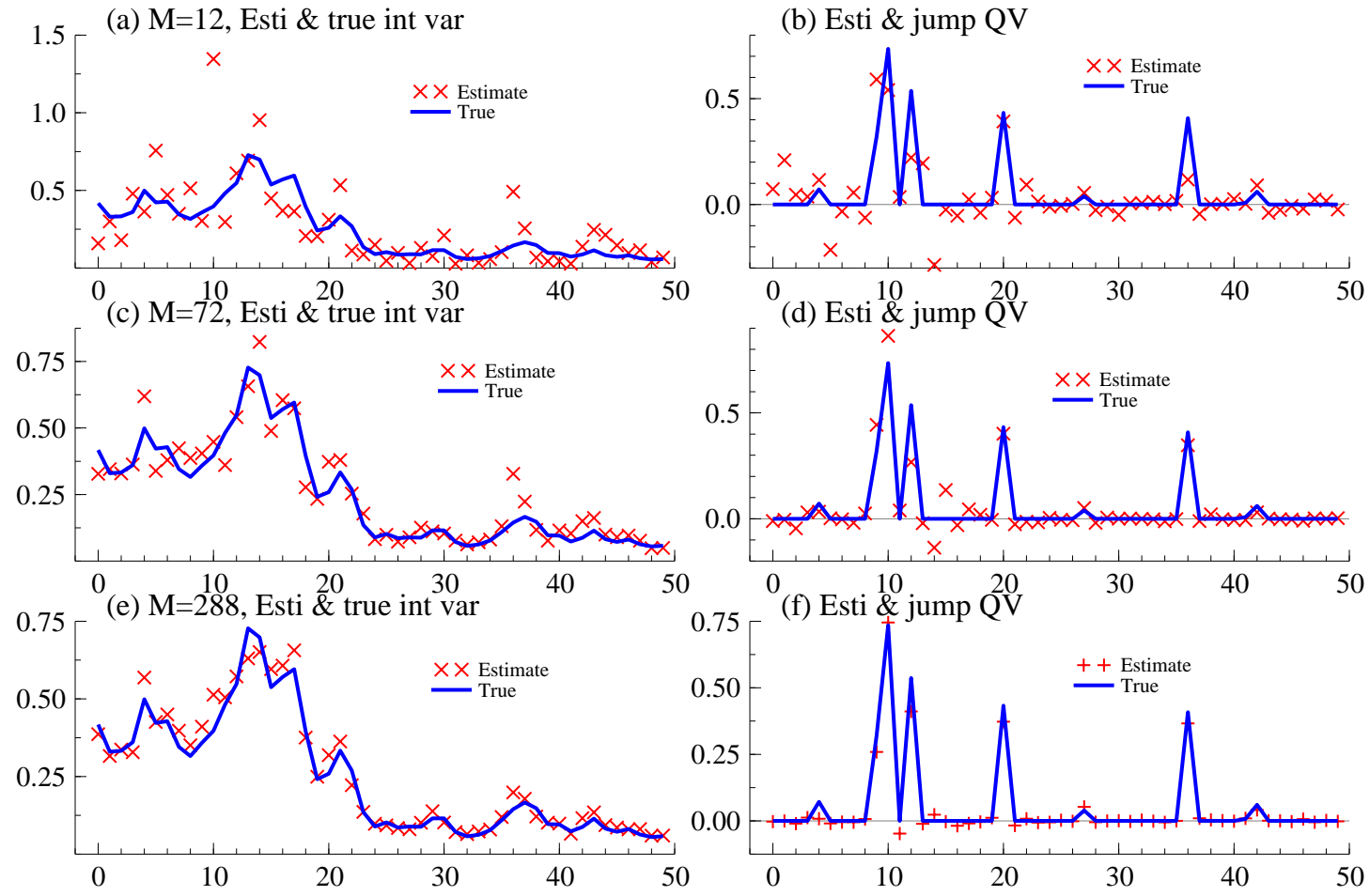

(d) Esti $\underset{\times}{\&}$ jump Q 20
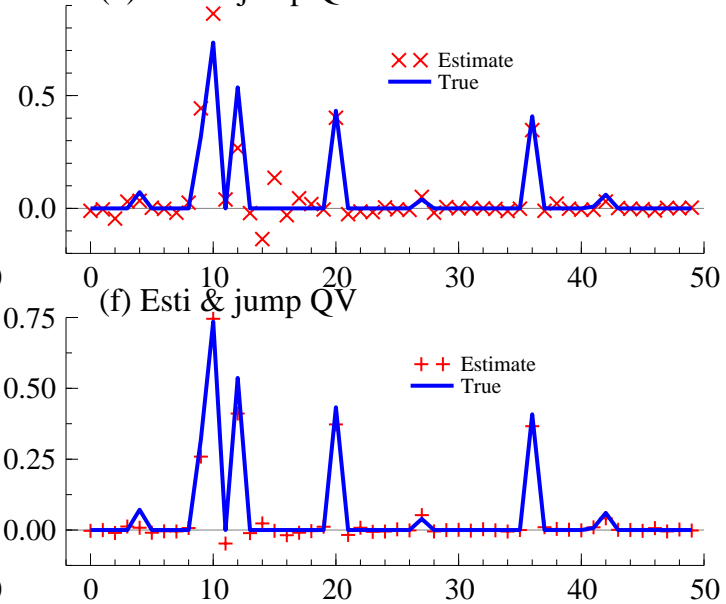

Figure 3: Simulation from a jump plus diffusion based $S V$ model, estimating the integrated variance and the $Q V$ of the jump components. Rows correspond to $M=12, M=72$ and $M=288$ respectively. (a), (c) and (e) plot the daily integrated variance $\sigma_{i}^{2}$ and the consistent estimator $\mu_{1}^{-2}\left\{y_{M}^{*}\right\}_{i}^{[1,1]}$. (b), (d) and (f) plot the $Q V$ of the jump process and the corresponding consistent estimator.

the realised variance error,

$$
\left[y^{(1) *}\right]_{i}-\mu_{1}^{-2}\left\{y_{M}^{*}\right\}_{i}^{[1,1]}=\sigma_{i}^{2}-\mu_{1}^{-2} \sum_{j=1}^{M-1}\left|y_{j, i}\right|\left|y_{j+1, i}\right|,
$$

the realised bipower variation error and, defining

$$
\widehat{\left[y_{M}^{(2) *}\right]_{i}^{[2]}}=\sum_{j=1}^{M} y_{j, i}^{2}-\mu_{1}^{-2} \sum_{j=1}^{M-1}\left|y_{j, i}\right|\left|y_{j+1, i}\right|
$$

we also examine

$$
\left[y^{(2) *}\right]_{i}-\left[\widehat{\left.y_{M}^{(2) *}\right]_{i}^{[2]}}=\left(\left[y^{*}\right]_{i}-\sigma_{i}^{2}\right)-\left(\sum_{j=1}^{M} y_{j, i}^{2}-\mu_{1}^{-2} \sum_{j=1}^{M-1}\left|y_{j, i}\right|\left|y_{j+1, i}\right|\right),\right.
$$

the realised jump error. The results are given in Table 1. This records the mean error, to see if the estimators are unbiased, as well as the 0.025 and 0.975 quantiles.

For each measure there is little bias even for small $M$. In the realised variance case the $95 \%$ range of the error falls quite quickly, although the interval is still substantial when $M=576$. An interesting feature is that the errors are roughly symmetric in this analysis. For the realised 


\begin{tabular}{|l|lll|lll|lll|}
\hline & \multicolumn{3}{|c|}{$\left[y^{*}\right]_{i}-\left[y_{M}^{*}\right]_{i}^{[2]}$} & \multicolumn{2}{c|}{$\left[y^{(1) *}\right]_{i}-\mu_{1}^{-2}$} & $\left\{y_{M}^{*}\right\}_{i}^{[1,1]}$ & \multicolumn{2}{c|}{$\left[y^{(2) *}\right]_{i}-\left[y_{M}^{(2) *}\right]_{i}^{[2]}$} \\
\hline & mean & .025 & .975 & mean & .025 & .975 & mean & 0.025 & 0.975 \\
\hline $\mathrm{M}=12$ & .001 & -.294 & .262 & -.000 & -.338 & .299 & .002 & -.179 & .168 \\
$\mathrm{M}=48$ & .001 & -.192 & .204 & -.001 & -.239 & .207 & .003 & -.097 & .129 \\
$\mathrm{M}=72$ & .001 & -.107 & .117 & -.000 & -.134 & .130 & .001 & -.067 & .075 \\
$\mathrm{M}=144$ & .000 & -.076 & .079 & -.001 & -.098 & .093 & .001 & -.043 & .057 \\
$\mathrm{M}=288$ & .000 & -.056 & .059 & -.000 & -.066 & .064 & .000 & -.033 & .037 \\
$\mathrm{M}=576$ & -.000 & -.043 & .041 & -.001 & -.048 & .043 & .000 & -.020 & .027 \\
\hline
\end{tabular}

Table 1: Finite sample behaviour of the estimators of the quadratic variation of log prices, integrated variance, and quadratic variation of the jump process. Reported are the errors. We calculate the mean value and the 0.025 and 0.975 quantiles of the sampling distribution.

bipower variation error the results are rather similar to the RV case. Interestingly the $95 \%$ interval is larger for this error than for the corresponding RV case. For the realised jump error, the $95 \%$ intervals are roughly symmetric.

\subsection{No jump case}

Interestingly, when we repeat the analysis, removing all jumps and comparing the performance of $\left[y_{M}^{*}\right]_{i}^{[2]}$ and $\mu_{1}^{-2}\left\{y_{M}^{*}\right\}_{i}^{[1,1]}$ as estimators of $\sigma_{i}^{2}$, we obtained the results given in Table 2 which indicate that the RV is slightly preferable in terms of accuracy. A theoretical confirmation of this result is indicated in Section 6 of this paper.

\begin{tabular}{|l|lll|lll|}
\hline & \multicolumn{3}{|c|}{$\left[y^{*}\right]_{i}-\left[y_{M}^{*}\right]_{i}^{[2]}$} & \multicolumn{3}{c|}{$\sigma_{i}^{2}-\mu_{1}^{-2}\left\{y_{M}^{*}\right\}_{i}^{[1,1]}$} \\
\hline & mean & .025 & .975 & mean & .025 & .975 \\
\hline $\mathrm{M}=12$ & .001 & -.284 & .258 & .002 & -.324 & .299 \\
$\mathrm{M}=48$ & .001 & -.189 & .201 & .000 & -.220 & .209 \\
$\mathrm{M}=72$ & .001 & -.106 & .113 & .001 & -.130 & .130 \\
$\mathrm{M}=144$ & .000 & -.074 & .079 & .000 & -.089 & .093 \\
$\mathrm{M}=288$ & -.000 & -.055 & .056 & .000 & -.061 & .064 \\
$\mathrm{M}=576$ & -.000 & -.042 & .040 & -.000 & -.046 & .043 \\
\hline
\end{tabular}

Table 2: Finite sample behaviour of the estimators of the quadratic variation of log prices and integrated variance in the case where there are no jumps. Reported are the errors. We calculate the mean value and the 0.025 and 0.975 quantiles of the sampling distribution.

\subsection{Improving the finite sample behaviour}

It is clear that

$$
\left[y^{(2) *}\right]_{i}=\left[y^{*}\right]_{i}-\sigma_{i}^{2}=\sum_{j=N\{\hbar(i-1)\}+1}^{N(\hbar i)} c_{j}^{2} \geq 0,
$$


while the estimator

$$
\widehat{\left[y_{M}^{(2) *}\right]_{i}^{[2]}}=\left[y_{M}^{*}\right]_{i}^{[2]}-\mu_{1}^{-2}\left\{y_{M}^{*}\right\}_{i}^{[1,1]}
$$

can be negative. Hence it would be sensible to sometimes replace it by the consistent estimator

$$
\max \left(\left[y_{M}^{*}\right]_{i}^{[2]}-\mu_{1}^{-2}\left\{y_{M}^{*}\right\}_{i}^{[1,1]}, 0\right)
$$

This would suggest the following estimator of the actual variance of the SV component

$$
\min \left\{\left[y_{M}^{*}\right]_{i}^{[2]}, \mu_{1}^{-2}\left\{y_{M}^{*}\right\}_{i}^{[1,1]}\right\} .
$$

Repeating the above experiment based on 5,000 days for the non-linear estimator of the QV of the jump component (23), we produce the analysis of errors given in Table 3 . We see that the finite sample behaviour of estimator improves quite considerably. Importantly, the latter term now has a very short right hand tail, which means we rarely overstate the presence of jumps by a large amount. Of course this estimator has the disadvantage of being biased.

\begin{tabular}{|l|lll|}
\hline & \multicolumn{3}{|c|}{ Jump error } \\
\hline & mean & .025 & .975 \\
\hline $\mathrm{M}=12$ & -.016 & -.179 & .025 \\
$\mathrm{M}=48$ & -.010 & -.097 & .017 \\
$\mathrm{M}=72$ & -.006 & -.067 & .011 \\
$\mathrm{M}=144$ & -.004 & -.043 & .011 \\
$\mathrm{M}=288$ & -.003 & -.033 & .008 \\
$\mathrm{M}=576$ & -.001 & -.020 & .006 \\
\hline
\end{tabular}

Table 3: Improved finite sample behaviour of the estimator of the quadratic variation of the jump process. Reported are the errors. We calculate the mean value and the $2.5 \%$ and $97.5 \%$ quantiles of the sampling distribution.

\section{$5 \quad$ Initial empirical work}

To illustrate some of the new theory, we return to the dataset discussed in the introduction. We now work with the full dataset from 1st December 1986 until 30th November 1996. All calculations will be based on $M=288$, that is we will employ 5 minute returns, calculating aggregate volatility statistics for each day within the sample.

Figure 4(a) shows the time series of the daily realised variance, while 4(b) shows the corresponding realised bipower variation. The difference between these two estimates, which is our estimate of the daily increments of QV of the jump component, is given in Figure 4(c). Finally, 4(d) shows the correlograms of the realised variances, the realised bipower variations and the estimated jump components of QV. The main features of Figure 4 is that the estimate of the 
(a) Daily realised variance

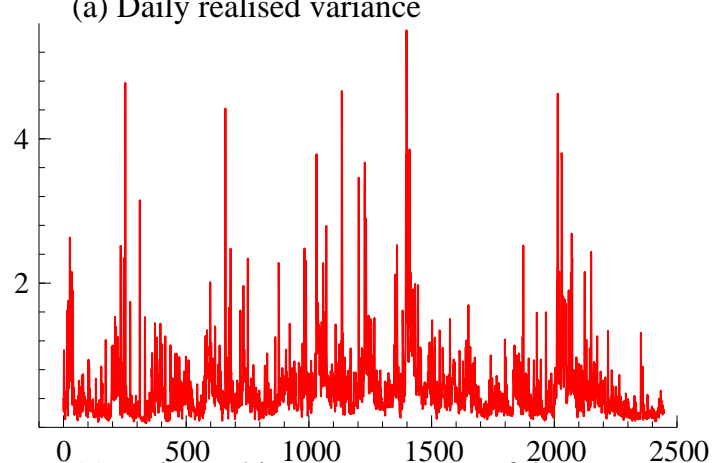

(c) Estimated jump component of Q 2000

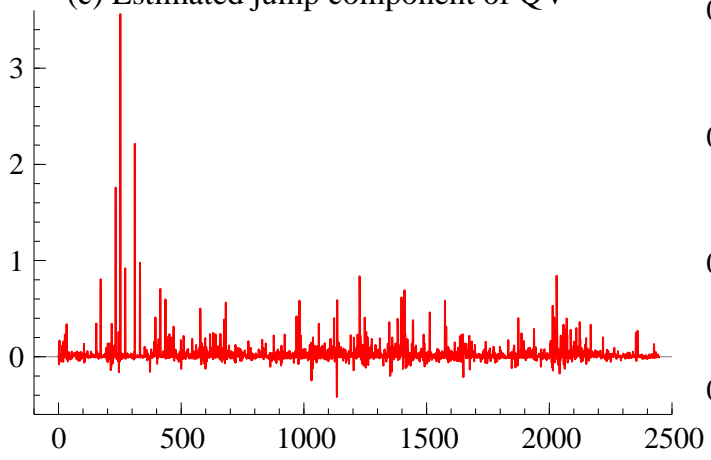

(b) Realised bipower variation
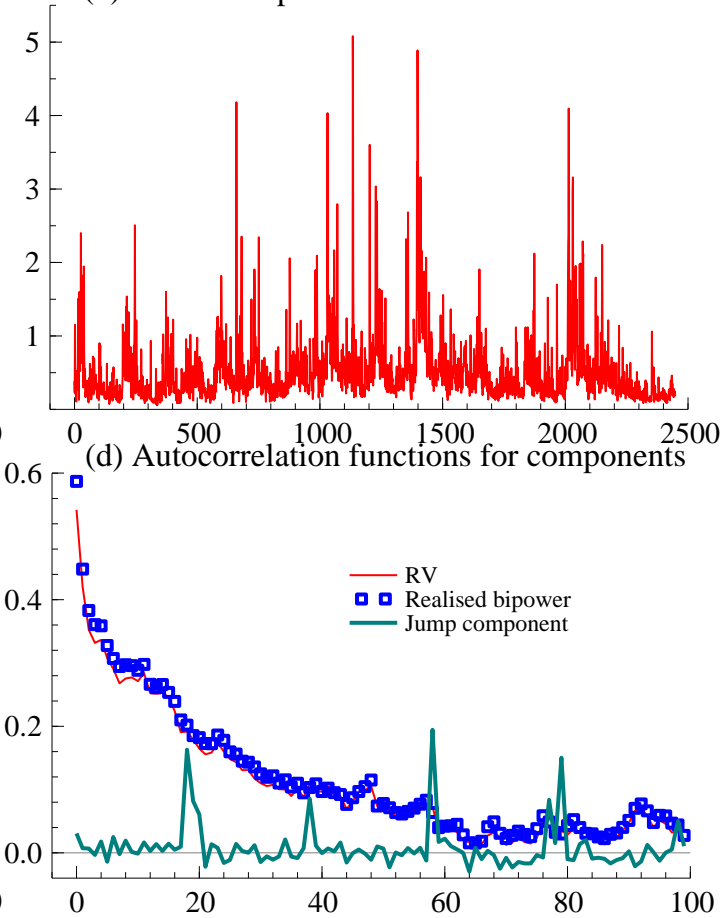

Figure 4: DM/dollar daily volatility measurements based on $M=288$.

integrated variance is the dominating component of the realised variance. Some of the estimates of the jump QV are quite large, especially at the start of the period. However, these jumps tend not to be very serially dependent. The serial dependence in the estimated integrated variances is larger than for the RVs. This is interesting, although the increase in dependence is quite modest. During this period, the average value of the jump component to daily QV is 0.0310 . Average realised variance is 0.528 , hence the size of the jump component is quite modest for the dataset.

Figure 5 focuses on two time-periods with large realised variances ${ }^{12}$. In both cases we look at 10 day periods of time, plotting in Figures 5(a) and (c) the log-price process every 5 minutes. Throughout Figures 5(a) and (c) we plot the prices using dots, rather than the more standard time series lines. In Figure 5(a) there is a large uptick in the price, with a movement of nearly two percent in a five minute period. This occurred on January 15th 1988. The Financial Times reported on its front page the next day "The dollar and share prices soared in hectic trading on world financial markets yesterday after the release of official figures showing that the US trade deficit had fallen to $\$ 13.22$ bn in November from October's record level of $\$ 17.63$ bn. The US currency surged 4 pfennigs and 4 yen within 10 minutes of the release of the figures

\footnotetext{
${ }^{12}$ The result does not change whether one uses the Andersen, Bollerslev, Diebold, and Labys (2001) version of this data, or the dataset which includes the Barndorff-Nielsen and Shephard (2002b) stochastic adjustments.
} 
(a) Path of the log-price process

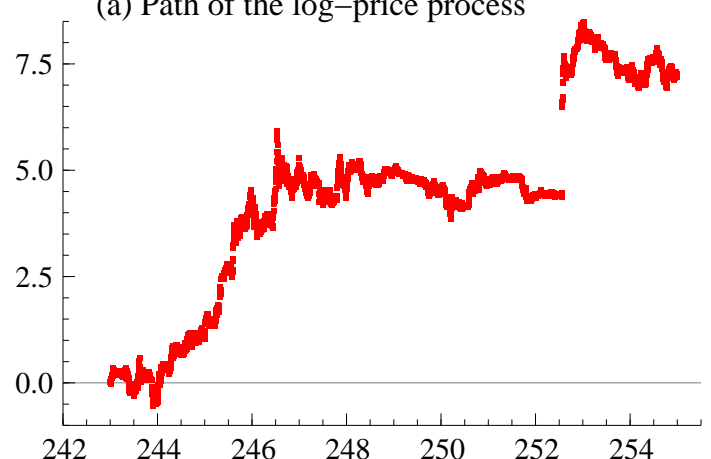

(c) Path of the log-price process

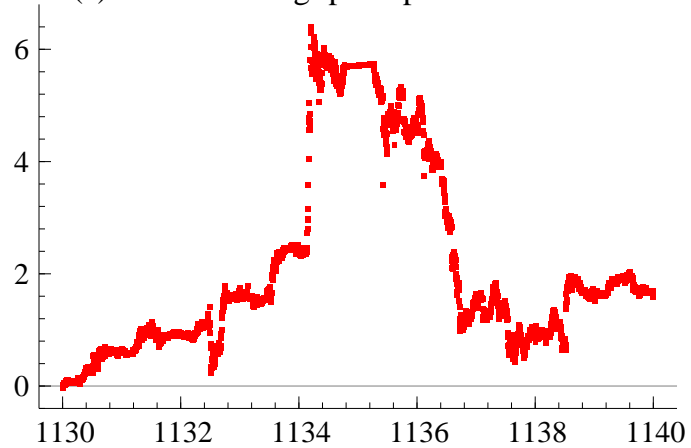

(b) RV and estimated IntVar

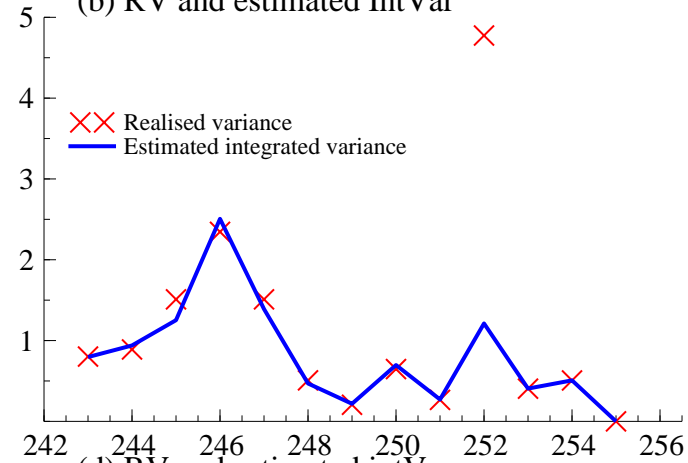

(d) RV and estimated intVar

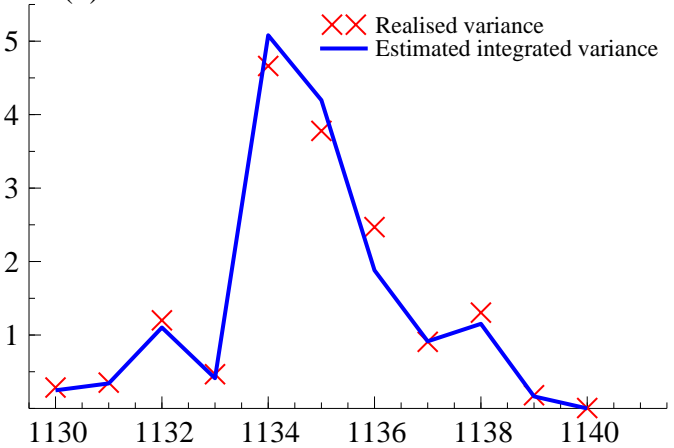

Figure 5: Two examples of small stretches of data with large realised variances. The log-prices are recorded every 5 minutes in (a) and (c). (a) has a large step change in the prices, which one may view as a jump. The change occured on 15th January 1988 when surprising U.S. balance of payment were announced. (c) has a number of large positive returns which are in sequence. This happened on August 19th 1991, the day of the coup against Gorbachev.

and maintained the day's highest levels in late New York business ... ." The 15th had a large realised variance, shown in $5(\mathrm{~b})$, but a much smaller estimate of the integrated variance. Hence the statistics are attributing a large component of the realised variance to the jump.

This effect contracts with Figure 5(c) where there is a three unit increase in the log-price, but this happens over around an hour long period with many positive returns. This happened on August 19th 1991. In the early hours of that day the Russian President Mikhail Gorbachev was overthrown after a coup by Communist hardliners. We can see in Figure 5(d) that the corresponding realised variance is very high, but so is the estimated integrated variance. Hence in this case the statistics have not flagged up a jump in the price, even though prices were moving rapidly in one direction.

\section{$6 \quad$ Extensions and discussion}

\subsection{Robust estimation of integrated covariance}

A vital concept in financial econometrics is covariance. We can formally base this on the concept of quadratic covariation. We will discuss this in the context of a bivariate semimartingale $\left(x^{*}, y^{*}\right)$. 
Then the quadratic covariation between $x^{*}$ and $y^{*}$ is

$$
\left[y^{*}, x^{*}\right](t)=\mathrm{p}-\lim _{M \rightarrow \infty} \sum_{j=1}^{M}\left\{y^{*}\left(t_{j}\right)-y^{*}\left(t_{j-1}\right)\right\}\left\{x^{*}\left(t_{j}\right)-x^{*}\left(t_{j-1}\right)\right\} .
$$

Note that using this notation

$$
\left[y^{*}, y^{*}\right](t)=\left[y^{*}\right](t)
$$

the QV of the $y^{*}$ process. Quadratic covariation has been recently studied in the econometric literature by, for example, Barndorff-Nielsen and Shephard (2002a) and Andersen, Bollerslev, Diebold, and Labys (2003).

In the context of multivariate $\mathcal{S} \mathcal{S} \mathcal{M}^{c}$ models (Barndorff-Nielsen and Shephard (2002a)) we assume

$$
\left(\begin{array}{c}
y^{*}(t) \\
x^{*}(t)
\end{array}\right)=\alpha^{*}(t)+m^{*}(t), \quad \text { where } m^{*}(t)=\int_{0}^{t} \Theta(u) \mathrm{d} w(u),
$$

$\alpha^{*} \in \mathcal{F} \mathcal{V}_{l o c}^{c}$, the elements of $\Theta$ are assumed càdlàg and $w$ is a vector of independent standard Brownian motions. We write $\Sigma(t)=\Theta(t) \Theta(t)^{\prime}$, and we need to make the additional assumption that $\int_{0}^{t} \Sigma_{k, l}(u) \mathrm{d} u<\infty, k, l=1,2$, to ensure that $m^{*}$ is a local martingale. This setup has the important property that

$$
\left[y^{*}, x^{*}\right](t)=\int_{0}^{t} \Sigma_{1,2}(u) d u,
$$

the integrated covariance of the price process. Andersen, Bollerslev, Diebold, and Labys (2003) emphasise the importance of estimating this type of term and they discuss the use of realised covariation to achieve this. A distribution theory for this estimator under the above type of model structure is given in Barndorff-Nielsen and Shephard (2002a).

An important property of quadratic variation is the so called polarisation result that

$$
\left[y^{*}+x^{*}\right](t)=\left[y^{*}\right](t)+\left[x^{*}\right](t)+2\left[y^{*}, x^{*}\right](t),
$$

which means that

$$
\left[y^{*}, x^{*}\right](t)=\frac{1}{2}\left\{\left[y^{*}+x^{*}\right](t)-\left[y^{*}\right](t)-\left[x^{*}\right](t)\right\} .
$$

Hence we can use realised bipower variation to consistently estimate $\left[y^{*}, x^{*}\right](t)$, or increments of it, by estimating each of the individual terms. Further, straightforwardly, this estimator is robust to jumps and we now have tools for assessing if the dependence between price processes is affected by the presence of jumps. We will explore this issue elsewhere in some detail.

\subsection{Generalisation to multipower variation}

We defined the bipower variation as

$$
p-\lim _{\delta \downarrow 0} \delta^{1-(r+s) / 2} \sum_{j=1}^{\lfloor t / \delta\rfloor-1}\left|y_{j}(t)\right|^{r}\left|y_{j+1}(t)\right|^{s}, \quad r, s \geq 0 .
$$


It is clear we can generalise this object by multiplying together a finite number of absolute returns raised to some non-negative power. In general we call this idea multipower variation. In particular the tripower variation process is

$$
\left\{y^{*}\right\}^{[r, s, u]}(t)=p-\lim _{\delta \downarrow 0} \delta^{1-(r+s+u) / 2} \sum_{j=1}^{\lfloor t / \delta\rfloor-2}\left|y_{j}(t)\right|^{r}\left|y_{j+1}(t)\right|^{s}\left|y_{j+2}(t)\right|^{u}, \quad r, s, u>0,
$$

while the quadpower variation process is

$$
\left\{y^{*}\right\}^{[r, s, u, v]}(t)=p-\lim _{\delta \downarrow 0} \delta^{1-(r+s+u+v) / 2} \sum_{j=1}^{\lfloor t / \delta\rfloor-3}\left|y_{j}(t)\right|^{r}\left|y_{j+1}(t)\right|^{s}\left|y_{j+2}(t)\right|^{u}\left|y_{j+3}(t)\right|^{v}, \quad r, s, u, v>0 .
$$

(a) $M=12$, Esti \& true int var

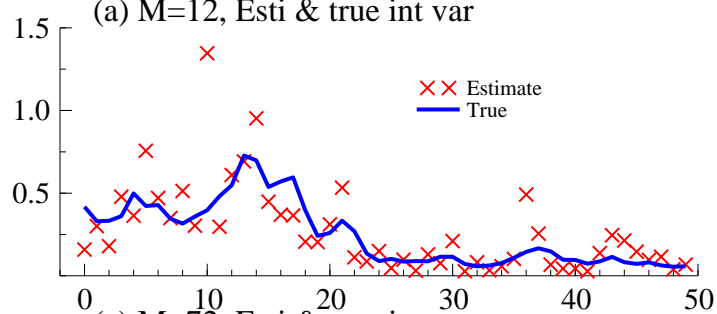

(c) $M=72$, Esti \& ${ }^{20}$ true int var
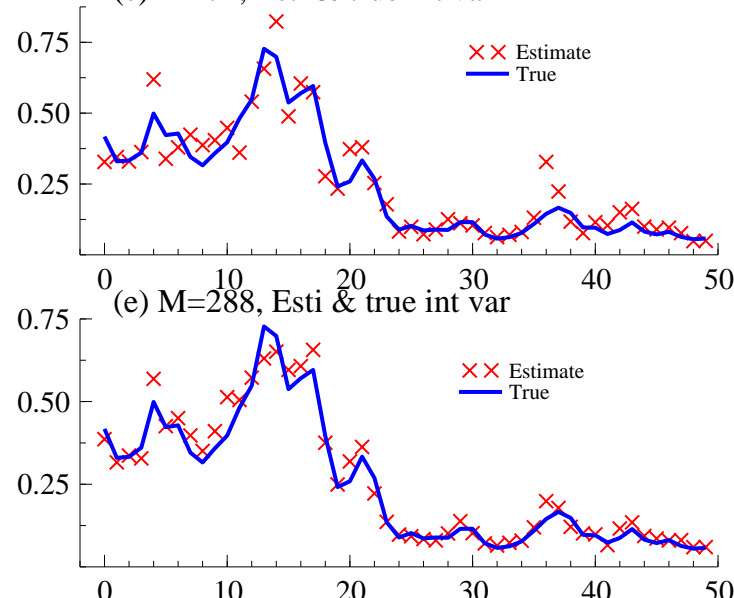

(b) Esti \& jump QV

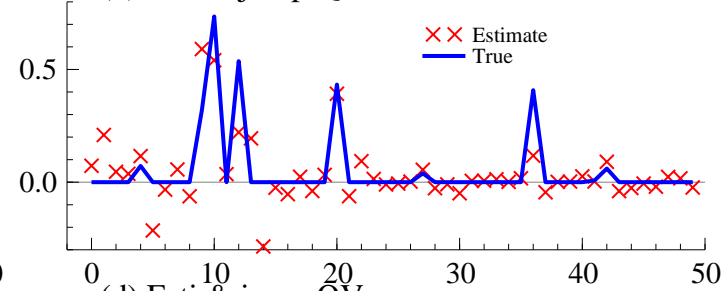

(d) Esti \& jump Q 20
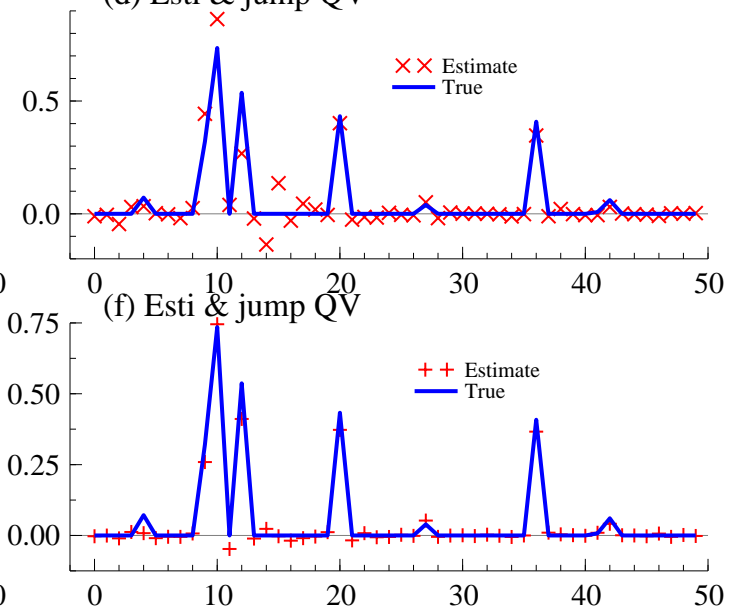

Figure 6: Estimation based on tripower variation. Simulation from a jump plus diffusion based $S V$ model. Rows correspond to $M=12, M=72$ and $M=288$ respectively. (a), (c) and (e) plot the daily integrated variance $\sigma_{i}^{2}$ and the consistent estimator $\mu_{2 / 3}^{-3}\left\{y_{M}^{*}\right\}_{i}^{[2 / 3,2 / 3,2 / 3]}$. (b), (d) and $(f)$ plot the $Q V$ of the jump component and the corresponding consistent estimator.

Example 1 Taking $r=s=u=2 / 3$, yields

$$
\mu_{2 / 3}^{-3}\left\{y^{*}\right\}^{[2 / 3,2 / 3,2 / 3]}(t)=\int_{0}^{t} \sigma^{2}(u) \mathrm{d} u,
$$

another new estimator of integrated variance. Figure 6 repeats the experiments reported in Figure 3 but replaces bipower with tripower. In particular we have plotted the time series of 
the realised tripower term

$$
\mu_{2 / 3}^{-3} \sum_{j=1}^{M-2}\left|y_{j, i}\right|^{2 / 3}\left|y_{j+1, i}\right|^{2 / 3}\left|y_{j+2, i}\right|^{2 / 3},
$$

instead of

$$
\mu_{1}^{-2} \sum_{j=1}^{M-1}\left|y_{j, i}\right|\left|y_{j+1, i}\right|
$$

Likewise

$$
\mu_{1 / 2}^{-4}\left\{y^{*}\right\}^{[1 / 2,1 / 2,1 / 2,1 / 2]}(t)=\int_{0}^{t} \sigma^{2}(u) \mathrm{d} u .
$$

This result is interesting as it is estimating integrated variance based on square roots of absolute returns, which is quite a robust item. Figure 7 again repeats the previous experiments, but now based on this quadpower concept. The realised version of the quadpower is

$$
\mu_{1 / 2}^{-4} \sum_{j=1}^{M-3} \sqrt{\left|y_{j, i} y_{j+1, i} y_{j+2, i} y_{j+3, i}\right|} .
$$

We see from Figure 7 that we do not produce very different answers. An important feature

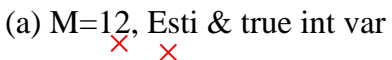
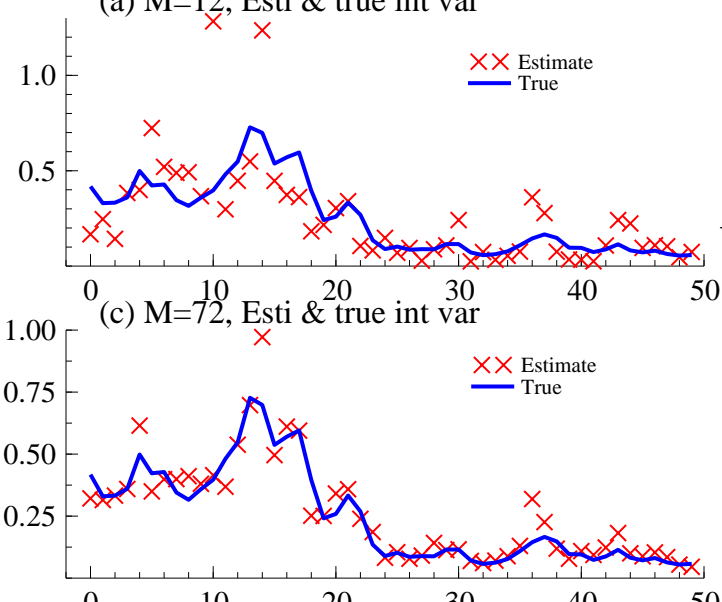

(e) $M=288$, Esti $\stackrel{20}{\&}$ true int var

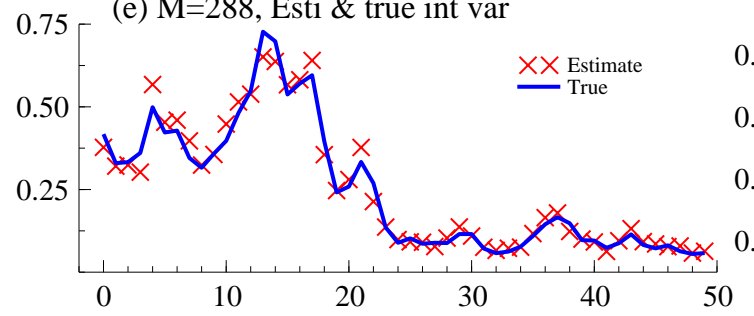

(b) Esti \& jump QV

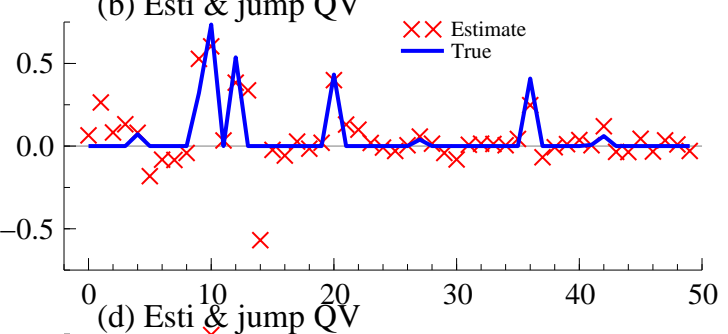

(d) Esti \& jump QV

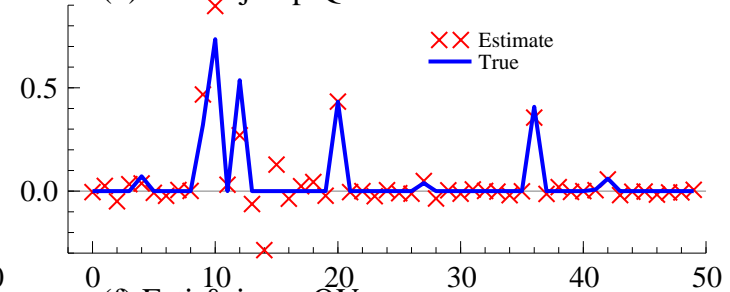

(f) Esti $\&$ jump QV

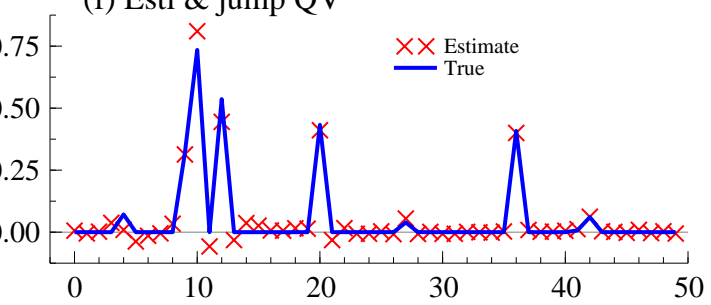

Figure 7: Estimation based on quadpower variation. Simulation from a jump plus diffusion based $S V$ model. Rows correspond to $M=12, M=72$ and $M=288$ respectively. (a), (c) and (e) plot the daily integrated variance $\sigma_{i}^{2}$ and the consistent estimator $\mu_{1 / 2}^{-4}\left\{y_{M}^{*}\right\}_{i}^{[1 / 2,1 / 2,1 / 2,1 / 2]}$. (b), (d) and $(f)$ plot the $Q V$ of the jump component and the corresponding consistent estimator.

of this graph is that for one period the estimate of the QV of the jump component is highly 
negative. This makes little sense, as we know the jump component has to be non-negative. We discussed this issue in the context of bipower in Section 4.3. If we use the same strategy here then we produce much more sensible results. They are plotted in Figure 8. This suggests that the estimators are reasonably accurate even for quite small samples.

(a) $M=12$, Esti \& true int var

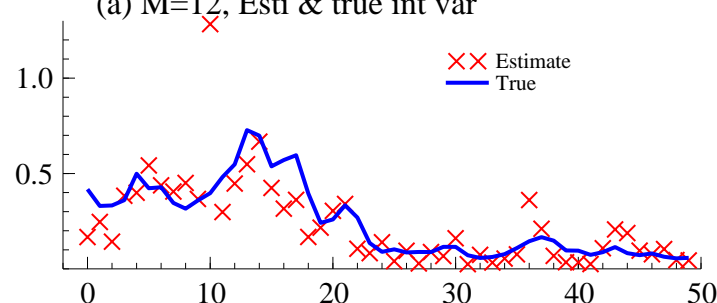

(c) $M=72$, Esti \& true int var

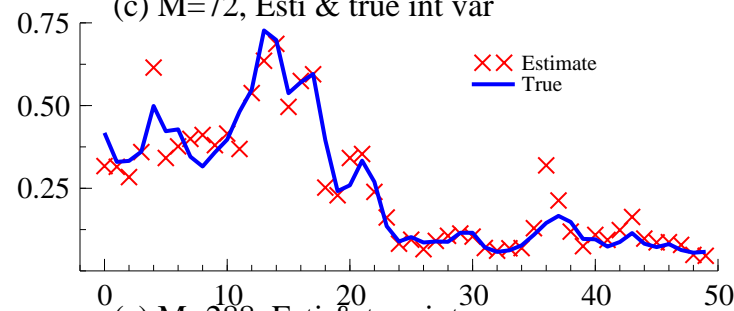

(e) $M=288$, Esti $\stackrel{20}{\&}$ true int var
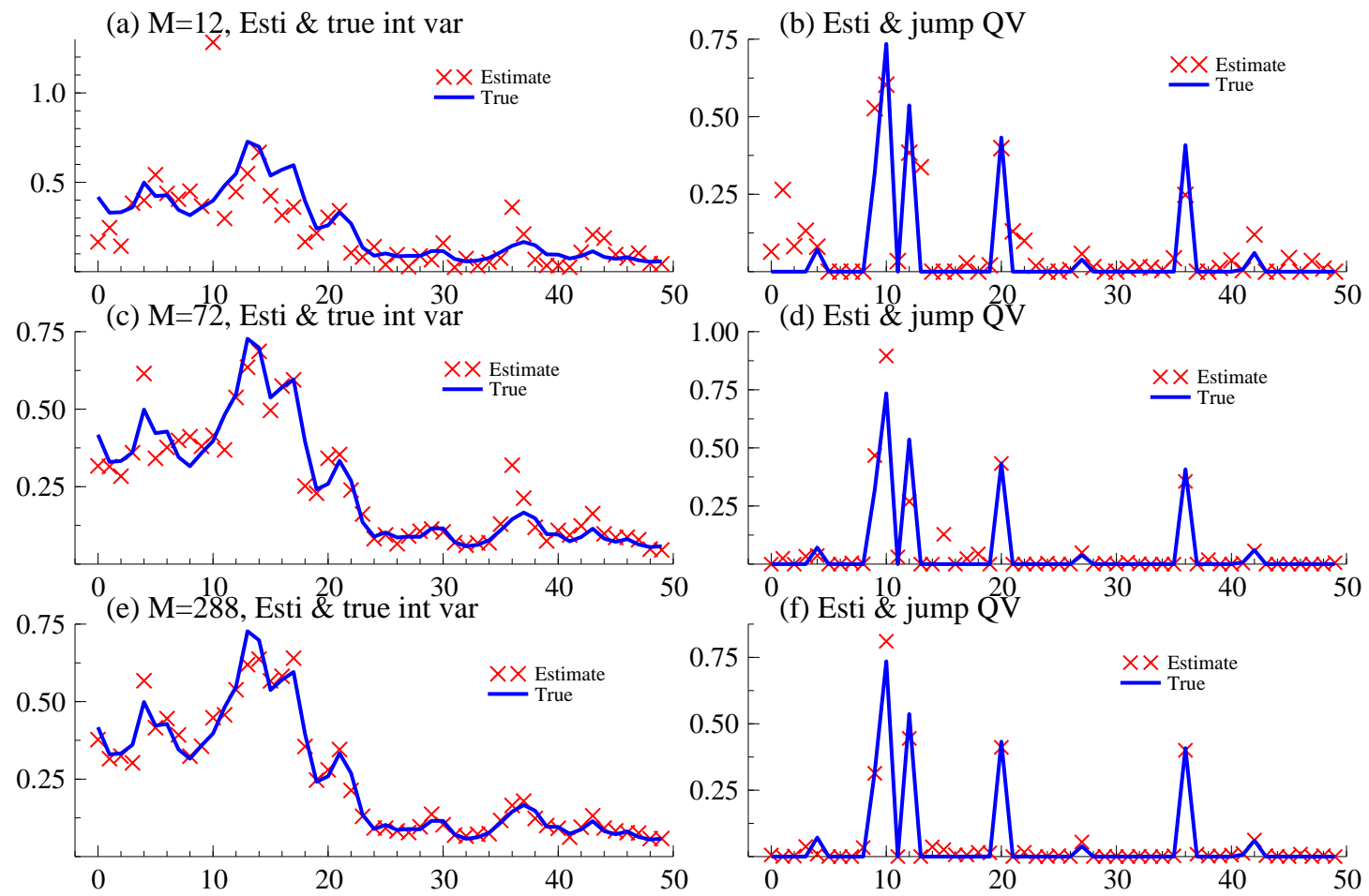

(d) Esti \& jump QV

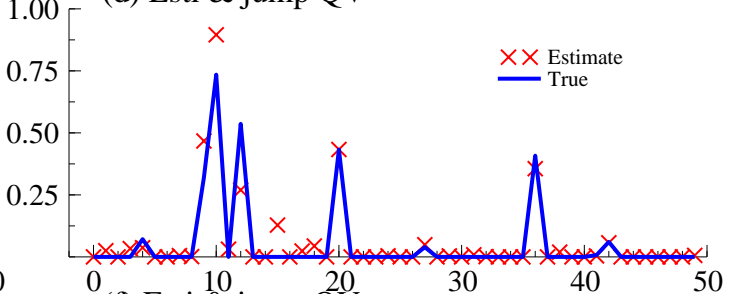

(f) Esti \& jump QV

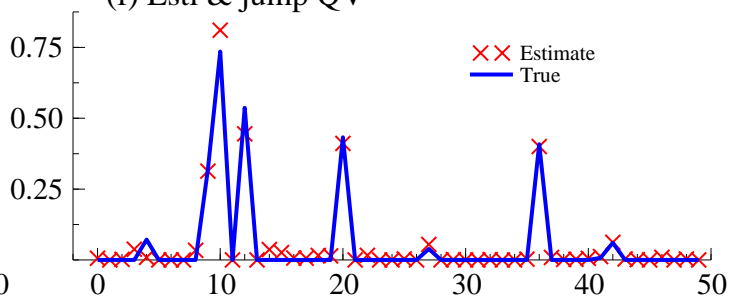

Figure 8: Estimation based on the truncated version of quadpower variation, which is enforced to improve the finite sample behaviour of the estimator. Simulation from a jump plus diffusion based $S V$ model. Rows correspond to $M=12, M=72$ and $M=288$ respectively. (a), (c) and (e) plot the daily integrated variance $\sigma_{i}^{2}$ and the consistent estimator. (b), (d) and (f) plot the jump $Q V$ and consistent estimator.

Example 2 Taking $r=s=2$, yields

$$
\left\{y^{*}\right\}^{[2,2]}(t)=\int_{0}^{t} \sigma^{4}(u) \mathrm{d} u,
$$

the integrated quarticity. The realised version of this was used by Barndorff-Nielsen and Shephard (2002a) to construct an asymptotic distribution theory for realised variance and covariation. A more robust object of this type is

$$
\mu_{1}^{-4}\left\{y^{*}\right\}^{[1,1,1,1]}(t)=\int_{0}^{t} \sigma^{4}(u) \mathrm{d} u .
$$

We will return to this in a moment when we discuss testing for jumps. 


\subsection{Some infinite activity Lévy processes}

In our analysis we have shown that the realised power variation of a compound Poisson process converges in probability to zero when the power is less than two. An interesting extension of this result is to Lévy processes with infinite numbers of jumps in finite time intervals. Then the question is whether bipower variation is robust to these types of jumps. Here we explore this problem, without giving a complete solution.

Recall Lévy processes are processes with independent and stationary increments. Examples of this type of process are the normal gamma (or variance gamma) process and the normal inverse Gaussian process. These are due to Madan and Seneta (1990) and Madan, Carr, and Chang (1998) in the former case and Barndorff-Nielsen (1997) and Barndorff-Nielsen (1998) in the latter case.

Let $z$ denote a Lévy process. It is characterised by the Lévy-Khintchine representation which says that we can write

$$
\log \mathrm{E} \exp \{i \zeta z(1)\}=a i \zeta-\frac{1}{2} \sigma^{2} \zeta^{2}-\int_{\mathbf{R}}\left\{1-e^{i \zeta x}+i \zeta x \mathbf{1}_{B}(x)\right\} W(\mathrm{~d} x),
$$

where $a \in R, \sigma \geq 0, B=[-1,1]$ and the Lévy measure $W$ must satisfy

$$
\int_{\mathbf{R}} \min \left\{1, x^{2}\right\} W(\mathrm{~d} x)<\infty
$$

and $W$ has no atom at 0 .

In this exposition we will assume the drift $a$ and the volatility $\sigma$ are zero, so $z$ is a pure jump process. The Lévy measure controls the jumps in the process. If $\int_{\mathbf{R}} W(\mathrm{~d} x)<\infty$ then the process is a compound Poisson process and so has a finite number of jumps. If this does not hold but $\int_{\mathbf{R}} \min \{1,|x|\} W(\mathrm{~d} x)<\infty$ then we say the process is of infinite activity finite variation (IAFV). It has an infinite number of jumps in any finite time interval. However, bounded variation feature of the process means that it can be decomposed into

$$
z(t)=z_{+}(t)-z_{-}(t)
$$

where $z_{+}$and $z_{-}$are independent subordinators. Recall a subordinator is a Lévy process with non-negative increments. Under the more general condition (25) this decomposition into positive and negative jumps is not possible.

We believe that the results of Section 3 extend to cases where the jump process is of the Lévy B-activity type and a discussion of this will be given elsewhere. Following the initial draft of this paper, Woerner (2003) has formally studied robustness results of this type, extending the derivations we have given to cases which allow infinite numbers of jumps in finite time periods. 


\subsection{Asymptotic distribution}

An important question is whether we can extend the convergence in probability result we saw for realised bipower variation to convergence in distribution. This type of result has been developed for realised variance by Barndorff-Nielsen and Shephard (2002b) and Barndorff-Nielsen and Shephard (2002a). It has been extended considerably in their work on realised power variation by Barndorff-Nielsen and Shephard (2003d) and Barndorff-Nielsen and Shephard (2003c). Following the initial draft of this paper, Barndorff-Nielsen and Shephard (2003a) have derived the joint asymptotic distribution of realised bipower and quadratic variation in the $r=s=1$ case, under some rather weak assumptions. Here we just outline the result, leaving the reader to look at that paper for technical conditions. In particular they show that

$$
\frac{\delta^{-1 / 2}}{\sqrt{\int_{0}^{t} \sigma^{4}(u) \mathrm{d} u}}\left(\begin{array}{l}
\sum_{j=1}^{\lfloor t / \delta\rfloor-1} y_{j}^{2}-\int_{0}^{t} \sigma^{2}(s) \mathrm{d} s \\
\mu_{1}^{-2} \sum_{j=1}^{\lfloor t / \delta\rfloor-1}\left|y_{j}\right|\left|y_{j+1}\right|-\int_{0}^{t} \sigma^{2}(s) \mathrm{d} s
\end{array}\right) \stackrel{\operatorname{law}}{\rightarrow} N\left\{0,\left(\begin{array}{ll}
2 & 2 \\
2 & 2.60907
\end{array}\right)\right\} .
$$

An implication is that the correlation between these two measures of integrated variance is constant through time and independent of the model. This analysis suggests that the realised variance statistic is, as expected, slightly more efficient than realised bipower variation. This agrees with the results in Table 2 . The correlation between the two statistics is approximately 0.875. The implication is that, under the $\mathcal{S} \mathcal{V} \mathcal{S} \mathcal{M}^{c}$ model, there is relatively little additional information in the realised bipower variation compared to the realised variance. Indeed, as

$$
\left(\begin{array}{ll}
2 & 2 \\
2 & 2.60907
\end{array}\right)^{-1}=\left(\begin{array}{cc}
2.1418 & -1.6418 \\
-1.6418 & 1.6418
\end{array}\right),
$$

the generalised least squares estimator of $\int_{0}^{t} \sigma^{2}(s) \mathrm{d} s$, based on the realised variance and realised bipower variation, will put weights on the realised variance and realised bipower variation which are approximately one and zero, respectively.

Straightforwardly we can also look at the behaviour of the difference between realised variance and realised bipower variation. The above limit theory immediately implies that

$$
\frac{\delta^{-1 / 2}}{\sqrt{\int_{0}^{t} \sigma^{4}(u) \mathrm{d} u}}\left(\sum_{j=1}^{\lfloor t / \delta\rfloor-1} y_{j}^{2}-\mu_{1}^{-2} \sum_{j=1}^{\lfloor t / \delta\rfloor-1}\left|y_{j}\right|\left|y_{j+1}\right|\right) \stackrel{\text { law }}{\rightarrow} N(0,0.6091) .
$$

This can be used to test for jumps, using the above as the null distribution. Clearly, it may be more desirable to look at the difference of the log of the realised variance and the realised 
bipower variation. Using the delta method on the multivariate distribution implies

$$
\frac{\delta^{-1 / 2}}{\sqrt{\frac{\int_{0}^{t} \sigma^{4}(u) \mathrm{d} u}{\left\{\int_{0}^{t} \sigma^{2}(u) \mathrm{d} u\right\}^{2}}}}\left\{\log \left(\sum_{j=1}^{\lfloor t / \delta\rfloor-1} y_{j}^{2}\right)-\log \left(\mu_{1}^{-2} \sum_{j=1}^{\lfloor t / \delta\rfloor-1}\left|y_{j}\right|\left|y_{j+1}\right|\right)\right\} \stackrel{\text { law }}{\rightarrow} N(0,0.6091) .
$$

Following the argument in Barndorff-Nielsen and Shephard (2004) we noted that in this type of context it is helpful to use Jensen's inequality which implies that

$$
\frac{\delta \mu_{1}^{-4} \sum_{j=1}^{\lfloor t / \delta\rfloor-3}\left|y_{j}\right|\left|y_{j+1}\right|\left|y_{j+2}\right|\left|y_{j+3}\right|}{\left\{\mu_{1}^{-2} \sum_{j=1}^{\lfloor t / \delta\rfloor-1}\left|y_{j}\right|\left|y_{j+1}\right|\right\}^{2}} \stackrel{p}{\rightarrow} \frac{\int_{0}^{t} \sigma^{4}(u) \mathrm{d} u}{\left\{\int_{0}^{t} \sigma^{2}(u) \mathrm{d} u\right\}^{2}} \geq t^{-1}
$$

This suggests using the asymptotic approximation

$$
\frac{\left\{\log \left(\sum_{j=1}^{\lfloor t / \delta\rfloor-1} y_{j}^{2}\right)-\log \left(\mu_{1}^{-2} \sum_{j=1}^{\lfloor t / \delta\rfloor-1}\left|y_{j}\right|\left|y_{j+1}\right|\right)\right\}}{\sqrt{0.6091 \max \left\{\frac{\delta}{t}, \sum_{j=1}^{\lfloor t / \delta\rfloor-3}\left|y_{j}\right|\left|y_{j+1}\right|\left|y_{j+2}\right|\left|y_{j+3}\right| /\left(\sum_{j=1}^{\lfloor t / \delta\rfloor-1}\left|y_{j}\right|\left|y_{j+1}\right|\right)^{2}\right\}}} \stackrel{\text { law }}{\rightarrow} N(0,1) .
$$

To illustrate the above theory we return to the Olsen data discussed above and use a onesided test to see if there are jumps in the series. Table 4 gives the percentage of rejections for the series, together with the associated critical value and significance levels. The Table shows there are around 1 in 5 rejections of the hypothesis of no jumps when we use a $5 \%$ significance level. This falls to around a 1 in 10 rejection when we move to a $1 \%$ level. Following the initial drafts of this paper, similar empirical results have been reported by Andersen, Bollerslev, and Diebold (2003) who have also studied the use of the difference between realised variance and bipower variation to test for jumps on this dataset. They have used such tests to then build reduced form models to improve upon existing forecasting devices for predicting future realised variances. Huang and Tauchen (2003) and Barndorff-Nielsen and Shephard (2003a) have independently and concurrently used simulations to study the finite sample performance of these types of tests, reaching broadly similar conclusions that transformed versions of the tests (such as the log version detailed above) are reasonably well behaved in empirically reasonable sample sizes.

\subsection{Other related work on jumps}

Here we briefly discuss some related econometric work which has studied jump type models.

There is a considerable literature on estimating continuous time parametric models. A particular focus has been on the class of SV plus jumps model. Johannes, Polson, and Stroud (2002) provide filtering methods for parameterised stochastic differential equations which exhibit 


\begin{tabular}{|lll|}
\hline Proportion of rejections & Critical value & Significance level \\
0.215 & 1.64 & 0.05 \\
0.119 & 2.32 & 0.01 \\
0.0600 & 3.09 & 0.001 \\
0.0383 & 3.71 & 0.0001 \\
\hline
\end{tabular}

Table 4: Olsen data, dollar against the DM. Tests for jumps based on asymptotic theory.

finite activity jumps. Their approach can be generalised to deal with infinite activity processes for it relies on the very flexible auxiliary particle filters introduced by Pitt and Shephard (1999), while corresponding filtering work on discrete time models was carried out by Chib, Nardari, and Shephard (2002). Andersen, Benzoni, and Lund (2002) and Chernov, Gallant, Ghysels, and Tauchen (2003) have used EMM methods to estimate and test some of these models. Maheu and McCurdy (2003) have constructed a jump/GARCH discrete time model for daily data which attempts to unscramble jumps and volatility changes, while Eraker, Johannes, and Polson (2003) look at the same issue based on SV models using Markov chain Monte Carlo methods (using the methods of handling diffusions introduced by Eraker (2001) and Elerian, Chib, and Shephard (2001)).

There is also a literature on discrete time parametric models of SV plus jumps. Chib, Nardari, and Shephard (2002) use simulation based likelihood inference which allows one to test for jumps in a standard manner. Other papers on this topic are pointed out in that paper's references.

Aït-Sahalia (2002) has recently asked the generic question of whether a continuous time Markov process exhibits jumps? He developed a theory for this based upon transition densities and then applied this to financial data. In some recent stimulating work Mancini (2001), Mancini (2003a) and Mancini (2003b) has tried to estimate the jump component of quadratic variation. She does this via the introduction of a jump threshold whose absolute value goes to zero as the number of observations within each day goes to infinity.

Finally, and closest to this paper, Barndorff-Nielsen and Shephard (2003b) have studied the second order properties of realised variance under the assumption that the local martingale component of prices is a time-changed Lévy process. Such models will have jumps in the price process except in the Brownian motion special case. For time-changed Lévy processes the realised variance is an inconsistent estimator of the increments of the time-change. They used their results to suggest simple quasi-likelihood estimators of these types of models. 


\section{Conclusion}

In this paper we have studied the recently formalised concept of realised power variation in the context of SV models where there can be occasional jumps. We have shown that sometimes the probability limit of these quantities are unaffected by rare jumps. Realised power variation has inspired us to introduce realised bipower variation. This shares the same robustness property, but can also be set up to estimate integrated power volatility in SV models. In a range of cases this produces an estimator of integrated variance in the presence of jumps. To our knowledge this is the first radical alternative to the commonly used realised variance estimator of this quantity. Importantly when we add jumps to the SV model the probability limit of the bipower estimator does not change, which means we can combine realised variance with realised bipower variation to estimate the quadratic variation of the jump component.

Various extensions of our work have been developed. We have outlined multivariate versions of the methods, a distribution theory and an assessment of the robustness to the $\alpha^{*}$ process. We think our paper may open up a number of interesting research avenues for econometricians.

\section{Acknowledgments}

We thank Eric Renault and René Garcia for asking us to give this paper as the invited address at the "Extremal Events in Finance" meeting in Montreal, October 25-26, 2002. Ole E. Barndorff-Nielsen's work is supported by CAF (www.caf.dk), which is funded by the Danish Social Science Research Council, and by MaPhySto (www.maphysto.dk), which is funded by the Danish National Research Foundation. Neil Shephard's research is supported by the UK's ESRC through the grants "Econometrics of trade-by-trade price dynamics" and "High frequency financial econometrics based upon power variation". All the calculations made in this paper are based on software written by the authors using the Ox language of Doornik (2001). We are in the debt of Xin Huang who pointed out an error in one of our arguments in an earlier version of the paper as well as giving detailed comments on other aspects of the paper. We have benefitted from conversations with Jeannette Woerner, Torben Andersen and Tim Bollerslev on this topic and the comments of Clive Bowsher and Carla Ysusi on an earlier version. Finally, we thank Elizabeth Martin, Nuffield College librarian, for various pieces of newspaper based research on the events we discuss in this paper. 


\section{References}

Aït-Sahalia, Y. (2002). Telling from discrete data whether the underlying continuous-time model is a diffusion. Journal of Finance 57, 2075-2112.

Andersen, T. G., L. Benzoni, and J. Lund (2002). An empirical investigation of continuoustime equity return models. Journal of Finance 57, 1239-1284.

Andersen, T. G. and T. Bollerslev (1997). Intraday periodicity and volatility persistence in financial markets. Journal of Empirical Finance 4, 115-158.

Andersen, T. G. and T. Bollerslev (1998a). Answering the skeptics: yes, standard volatility models do provide accurate forecasts. International Economic Review 39, 885-905.

Andersen, T. G. and T. Bollerslev (1998b). Deutsche mark-dollar volatility: intraday activity patterns, macroeconomic announcements, and longer run dependencies. Journal of Finance 53, 219-265.

Andersen, T. G., T. Bollerslev, and F. X. Diebold (2003). Some like it smooth, and some like it rough: untangling continuous and jump components in measuring, modeling and forecasting asset return volatility. Unpublished paper: Economics Dept, Duke University.

Andersen, T. G., T. Bollerslev, and F. X. Diebold (2004). Parametric and nonparametric measurement of volatility. In Y. Ait-Sahalia and L. P. Hansen (Eds.), Handbook of Financial Econometrics. Amsterdam: North Holland. Forthcoming.

Andersen, T. G., T. Bollerslev, F. X. Diebold, and P. Labys (2001). The distribution of exchange rate volatility. Journal of the American Statistical Association 96, 42-55. Correction published in 2003, volume 98, page 501.

Andersen, T. G., T. Bollerslev, F. X. Diebold, and P. Labys (2003). Modeling and forecasting realized volatility. Econometrica 71, 579-625.

Andreou, E. and E. Ghysels (2002). Rolling-sampling volatility estimators: some new theoretical, simulation and empirical results. Journal of Business and Economic Statistics 20, 363-376.

Andreou, E. and E. Ghysels (2003). The impact of sampling frequency and volatility estimators on change-point tests. Unpublished paper: Department of Economics, University of North Carolina, Chapel Hill.

Barndorff-Nielsen, O. E. (1997). Normal inverse Gaussian distributions and stochastic volatility modelling. Scandinavian Journal of Statistics 24, 1-14. 
Barndorff-Nielsen, O. E. (1998). Processes of normal inverse Gaussian type. Finance and Stochastics 2, 41-68.

Barndorff-Nielsen, O. E., S. E. Graversen, and N. Shephard (2003). Power variation and stochastic volatility: a review and some new results. Journal of Applied Probability. Forthcoming. Festschrift for C.C. Heyde.

Barndorff-Nielsen, O. E. and N. Shephard (2001). Non-Gaussian Ornstein-Uhlenbeck-based models and some of their uses in financial economics (with discussion). Journal of the Royal Statistical Society, Series B 63, 167-241.

Barndorff-Nielsen, O. E. and N. Shephard (2002a). Econometric analysis of realised covariation: high frequency covariance, regression and correlation in financial economics. Unpublished paper: Nuffield College, Oxford, Economics working paper 2002-W13.

Barndorff-Nielsen, O. E. and N. Shephard (2002b). Econometric analysis of realised volatility and its use in estimating stochastic volatility models. Journal of the Royal Statistical Society, Series B 64, 253-280.

Barndorff-Nielsen, O. E. and N. Shephard (2003a). Econometrics of testing for jumps in financial economics using bipower variation. Unpublished discussion paper: Nuffield College, Oxford.

Barndorff-Nielsen, O. E. and N. Shephard (2003b). Impact of jumps on returns and realised variances: econometric analysis of time-deformed Lévy processes. Unpublished paper: Nuffield College, Oxford.

Barndorff-Nielsen, O. E. and N. Shephard (2003c). Power variation and time change. Unpublished paper: Nuffield College, Oxford.

Barndorff-Nielsen, O. E. and N. Shephard (2003d). Realised power variation and stochastic volatility. Bernoulli 9, 243-265. Correction is available at www.levyprocess.org.

Barndorff-Nielsen, O. E. and N. Shephard (2004). How accurate is the asymptotic approximation to the distribution of realised volatility? In D. W. K. Andrews, J. Powell, P. A. Ruud, and J. H. Stock (Eds.), Identification and Inference for Econometric Models. A Festschrift in Honour of T.J. Rothenberg. Cambridge: Cambridge University Press. Forthcoming.

Black, F. (1976). Studies of stock price volatility changes. Proceedings of the Business and Economic Statistics Section, American Statistical Association, 177-181.

Chernov, M., A. R. Gallant, E. Ghysels, and G. Tauchen (2003). Alternative models of stock price dynamics. Journal of Econometrics 116, 225-257. 
Chib, S., F. Nardari, and N. Shephard (2002). Markov chain Monte Carlo methods for generalized stochastic volatility models. Journal of Econometrics 108, 281-316.

Cox, J. C., J. E. Ingersoll, and S. A. Ross (1985). A theory of the term structure of interest rates. Econometrica 53, 385-407.

Dacorogna, M. M., R. Gencay, U. A. Müller, R. B. Olsen, and O. V. Pictet (2001). An Introduction to High-Frequency Finance. San Diego: Academic Press.

Dacorogna, M. M., U. A. Müller, R. B. Olsen, and O. V. Pictet (1998). Modelling short term volatility with GARCH and HARCH. In C. Dunis and B. Zhou (Eds.), Nonlinear Modelling of High Frequency Financial Time Series, pp. 161-176. Chichester: Wiley.

Doob, J. L. (1953). Stochastic Processes. New York: John Wiley and Sons.

Doornik, J. A. (2001). Ox: Object Oriented Matrix Programming, 3.0. London: Timberlake Consultants Press.

Elerian, O., S. Chib, and N. Shephard (2001). Likelihood inference for discretely observed non-linear diffusions. Econometrica 69, 959-993.

Eraker, B. (2001). Markov chain Monte Carlo analysis of diffusion models with application to finance. Journal of Business and Economic Statistics 19, 177-191.

Eraker, B., M. Johannes, and N. G. Polson (2003). The impact of jumps in returns and volatility. Journal of Finance 53, 1269-1300.

Ghysels, E., A. C. Harvey, and E. Renault (1996). Stochastic volatility. In C. R. Rao and G. S. Maddala (Eds.), Statistical Methods in Finance, pp. 119-191. Amsterdam: North-Holland.

Harvey, A. C., E. Ruiz, and N. Shephard (1994). Multivariate stochastic variance models. Review of Economic Studies 61, 247-264.

Heston, S. L. (1993). A closed-form solution for options with stochastic volatility, with applications to bond and currency options. Review of Financial Studies 6, 327-343.

Huang, X. and G. Tauchen (2003). The relative contribution of jumps to total price variation. Unpublished paper: Department of Economics, Duke University.

Jacod, J. and A. N. Shiryaev (1987). Limit Theorems for Stochastic Processes. Springer-Verlag: Berlin.

Johannes, M., N. G. Polson, and J. Stroud (2002). Nonlinear filtering of stochastic differential equations with jumps. Unpublished paper: Graduate School of Business, Columbia University. 
Karatzas, I. and S. E. Shreve (1991). Brownian Motion and Stochastic Calculus (2 ed.), Volume 113 of Graduate Texts in Mathematics. Berlin: Springer-Verlag.

Kim, S., N. Shephard, and S. Chib (1998). Stochastic volatility: likelihood inference and comparison with ARCH models. Review of Economic Studies 65, 361-393.

Lépingle, D. (1976). La variation d'ordre p des semi-martingales. Zeitschrift fur Wahrscheinlichkeitstheorie und verwandte Gebiete 36, 295-316.

Lyons, T. (1994). Differential equations driven by rough signals. I. An extension of an inequality by L.C.Young. Mathematical Research Letters 1, 451-464.

Lyons, T. J. and Z. Qian (2002). System Control and Rough Paths. Oxford: Oxford University Press.

Madan, D. B., P. Carr, and E. Chang (1998). The variance gamma process and option pricing. European Finance Review 2, 79-105.

Madan, D. B. and E. Seneta (1990). The VG model for share market returns. Journal of Business 63, 511-524.

Maheswaran, S. and C. A. Sims (1993). Empirical implications of arbitrage-free asset markets. In P. C. B. Phillips (Ed.), Models, Methods and Applications of Econometrics, pp. 301-316. Basil Blackwell.

Maheu, J. M. and T. H. McCurdy (2003). News arrival, jump dynamics and volatility components for individual stock returns. Journal of Finance. Forthcoming.

Mancini, C. (2001). Does our favourite index jump or not. Dipartimento di Matematica per le Decisioni, Universita di Firenze.

Mancini, C. (2003a). Estimation of the characteristics of jump of a general Poisson-diffusion process. Dipartimento di Matematica per le Decisioni, Universita di Firenze.

Mancini, C. (2003b). Statistics of a Poisson-Gaussian process. Dipartimento di Matematica per le Decisioni, Universita di Firenze.

Merton, R. C. (1976). Option pricing when underlying stock returns are discontinuous. Journal of Financial Economics 3, 125-144.

Mikosch, T. and R. Norvaiša (2000). Stochastic integral equations without probability. Bernoulli 6, 401-434.

Nelson, D. B. (1991). Conditional heteroskedasticity in asset pricing: a new approach. Econometrica 59, 347-370. 
Pitt, M. K. and N. Shephard (1999). Filtering via simulation: auxiliary particle filter. Journal of the American Statistical Association 94, 590-599.

Poterba, J. and L. Summers (1986). The persistence of volatility and stock market fluctuations. American Economic Review 76, 1124-1141.

Schwert, G. W. (1989). Why does stock market volatility change over time? Journal of Finance 44, 1115-1153.

Shephard, N. (1996). Statistical aspects of ARCH and stochastic volatility. In D. R. Cox, D. V. Hinkley, and O. E. Barndorff-Nielsen (Eds.), Time Series Models in Econometrics, Finance and Other Fields, pp. 1-67. London: Chapman \& Hall.

Shiryaev, A. N. (1999). Essentials of Stochastic Finance: Facts, Models and Theory. Singapore: World Scientific.

Taylor, S. J. (1994). Modelling stochastic volatility. Mathematical Finance 4, 183-204.

Woerner, J. (2002). Variational sums and power variation: a unifying approach to model selection and estimation in semimartingale models. Statistics and Decisions 21, 47-68.

Woerner, J. (2003). Estimation of integrated volatility in stochastic volatility models. Unpublished paper: OCIAM, Mathematical Institute, University of Oxford. 